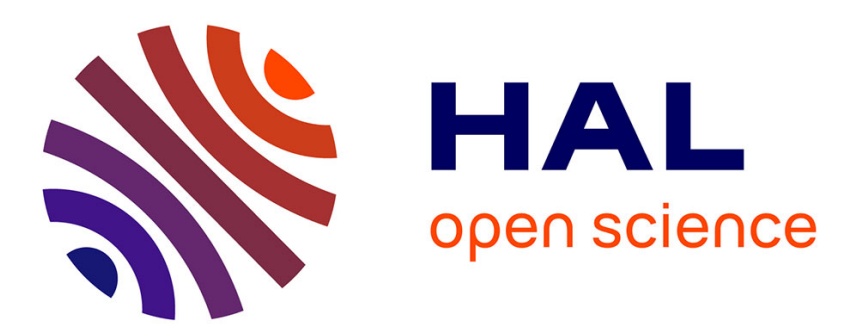

\title{
Multiparameter identification of a lossy fluid-like object from its transient acoustic response
}

\author{
Thierry Scotti, Armand Wirgin
}

\section{To cite this version:}

Thierry Scotti, Armand Wirgin. Multiparameter identification of a lossy fluid-like object from its transient acoustic response. Inverse Problems in Science and Engineering, 2014, 10.1080/17415977.2013.867485 . hal-00801081

\section{HAL Id: hal-00801081 \\ https://hal.science/hal-00801081}

Submitted on 15 Mar 2013

HAL is a multi-disciplinary open access archive for the deposit and dissemination of scientific research documents, whether they are published or not. The documents may come from teaching and research institutions in France or abroad, or from public or private research centers.
L'archive ouverte pluridisciplinaire HAL, est destinée au dépôt et à la diffusion de documents scientifiques de niveau recherche, publiés ou non, émanant des établissements d'enseignement et de recherche français ou étrangers, des laboratoires publics ou privés. 


\title{
Multiparameter identification of a lossy fluid-like object from its transient acoustic response
}

\author{
Thierry Scotti ${ }^{*}$ Armand Wirgin ${ }^{\dagger}$
}

March 14, 2013

\begin{abstract}
The Nelder-Mead downhill Simplex optimization method is employed for multiparameter retrieval in connection with a transient acoustic wave canonical inverse problem in both the inverse crime and non-inverse crime settings. In the inverse crime setting, it is shown that the Simplex scheme may require a large number of iterations and function calls to converge to the (unique) target solution, defined by a simple zerothorder (i.e., min min cost) regularization. In the non-inverse crime situation, in which a fixed prior employed in the retrieval model is different from its true counterpart, three types of regularization strategies: zeroth-order, first-order (i.e., a retrieved geometrical parameter must be non-negative, followed by min min cost), second-order (i.e., all retrieved parameters must be within their respective initial-brackets, followed by min min cost), are employed to obtain a unique solution. Only the second-order regularization scheme is found to be suitable to obtain stable results. Retrieval errors are computed, and found to be large, for various non-inverse crime scenarios.
\end{abstract}

*LMA, CNRS, UPR 7051, Aix-Marseille Univ, Centrale Marseille, F-13402 Marseille Cedex 20, France

${ }^{\dagger}$ LMA, CNRS, UPR 7051, Aix-Marseille Univ, Centrale 15Marseille, F-13402 Marseille Cedex 20, France 


\section{Contents}

1 Introduction $\quad 4$

2 Ingredients of multiparameter inversion of time- domain data 5

2.1 The two models . . . . . . . . . . . . . . . . . . . . . 5

2.2 Basic retrieval scheme . . . . . . . . . . . . . . . . 5

2.3 The inverse crime . . . . . . . . . . . . . . . . . 5

2.4 Minimization of the cost functional . . . . . . . . . . . . . . . . . . . . . . . . . 6

2.5 The Simplex algorithm . . . . . . . . . . . . . . . . 6

2.6 Parameter retrieval schemes . . . . . . . . . . . . . . . . . 7

2.7 The retrieval error generated by prior uncertainty . . . . . . . . . . . . 8

3 The models for computing the signals $\mathfrak{s}\left(\mathrm{x}_{j}, t_{k}, \mathbf{q}, \mathbf{r}\right)$ and $\mathfrak{S}\left(\mathrm{x}_{j}, t_{k}, \mathbf{Q}, \mathbf{R}\right) \quad 8$

3.1 Model description . . . . . . . . . . . . . . . . . 8

3.2 Acoustic field solutions . . . . . . . . . . . . . . . . . . . . 9

4 Numerical issues $\quad 11$

5 The parameters involved in the simulated data and retrieval data $\quad 12$

5.1 The parameters involved in the (simulated) data . . . . . . . . . . . . 12

5.2 The parameters involved in the retrieval data . . . . . . . . . . . . 13

6 Numerical results for the inverse crime situation $\quad 14$

6.1 Parameters and conventions common to the figures . . . . . . . . . . . . . 14

6.1.1 A rule for spotting admissible solutions . . . . . . . . . . . . . 15

6.2 A first result analogous to those of Buchanan et al. [5] . . . . . . . . . 15

6.2.1 The Buchanan et al. procedure . . . . . . . . . . . . . . . 15

6.2 .2 Our procedure . . . . . . . . . . . . . . . . 16

6.2.3 Comments on the Buchanan et al. procedure . . . . . . . . . . . . . 19

6.3 Subsequent results that go beyond the one analogous to those of Buchanan,

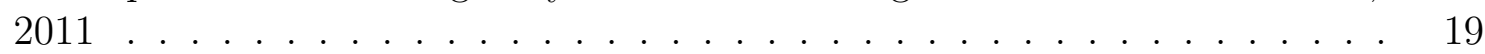

6.4 Effect of the variation of $M F E$ for a large initial parameter interval $d_{R_{j}}=$

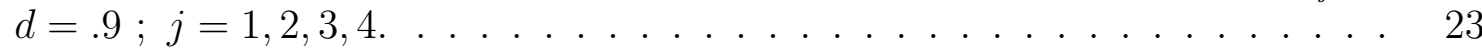

6.4.1 Comments on figs. $7-9 \ldots \ldots \ldots \ldots$. . . . . . . . . . . . . 27

6.5 Indications of the manner in which the retrievals depend on the choice of excitation and sensor parameters . . . . . . . . . . . . 27

6.5.1 Effect of the variation of the excitation parameter $\alpha=\mathcal{A} \ldots \ldots$. . . 27

6.5.2 Effect of the variation of sensor parameter $b=B \ldots \ldots . . . . .29$

6.5.3 Effect of the variation of sensor parameter $N_{\theta} \ldots \ldots$. . . . . . 30

6.6 Final comments on the numerical results for the inverse crime situation . . . 31 
7 Numerical results for the non-inverse crime situation in which there is an uncertainty on the prior relative to the wavespeed in the host medium 31

7.1 Effect of the variation of $M F E \ldots \ldots \ldots \ldots \ldots$

7.1.1 Initial parameter intervals corresponding to $d_{R_{j}}=d=.5 ; j=1,2,3,4$. 32

7.1.2 Initial parameter intervals corresponding to $d_{R_{j}}=d=.9 ; j=1,2,3,4$. 34

7.1.3 Comments on figs. 13-16 . . . . . . . . . . . . . . . 36

7.2 Variation of $d$ for larger discordances between $C^{[0]}$ and $c^{[0]} \ldots \ldots \ldots 36$

7.3 Effect of $C^{[0]}$ prior uncertainty on retrieval error . . . . . . . . . . . 38

7.3.1 The revised graph of retrieved parameters versus $\mathcal{C}^{[0]} \ldots \ldots \ldots$

8 Numerical results concerning the effect of the other prior uncertainties on retrieval error

8.1 Effect of $\mathcal{R}^{[0]}$ prior uncertainty $\ldots \ldots \ldots \ldots \ldots$

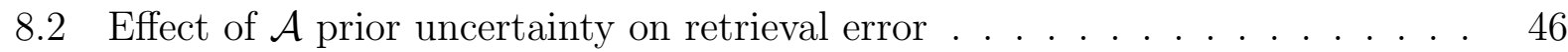

8.3 Effect of $\mathcal{B}$ prior uncertainty . . . . . . . . . . . . . . . . . . . 47

8.4 Effect of $\Theta^{i}$ prior uncertainty $\ldots \ldots \ldots \ldots \ldots$

9 Conclusions 49 


\section{Introduction}

The ultimate goal of the majority of the applications of inverse scattering is to obtain a quantitative image [21] of a corrupted (such as by a crack: [1],[2], [39]), diseased (such as affected by a tumor: [34], [48], [33], [16], [46], [19]), or otherwise inhomogeneous ([32], [24], [14], [9], [25]) body. To do this in optimal manner (e.g., via a domain integral approach: [14], [48]) requires the previous knowledge of the (termed 'specific' in [43], 'macro model' in [11], [13], [41], 'background' in [8]) Green's function of the corresponding uncorrupted, healthy ([27], [14]), or homogeneous body. The determination of this Green's function requires quantitative information about the size, shape and constitutive parameters of the healthy body, which must be determined by solving another inverse (scattering or vibration) problem [28]. If the diseased body is a cylinder with a tumor, then the healthy body is the same cylinder without the tumor; this cylinder is the object we wish to characterize herein.

We revisit an oft-studied acoustically-solicited (analogous to the electromagnetic wave: [24], [25]) configuration involving a canonical object (simple circular shape, homogeneous viscous fluid (analogous to lossy dielectric [12], [19]) composition), thereby:

- enabling the direct-scattering problem to be solved in a quasi-analytic manner (e.g., [31], [43]), and

- enabling nonlinear full (transient)- wave inversions (e.g., [38], [41], [37], [29]) to be carried out in a reasonable amount of time.

Numerous numerical tests of the multiparameter retrieval (such as in [28],[35] (there is a sign error on $c^{[1] i}$ in this paper)) are made herein in the hope of shedding light on the crucial aspects, notably concerning regularization strategies and retrieval error, of more-complicated (i.e., involving objects with non-canonical shapes and/or that are inhomogeneous) transientwave inverse problems.

In particular, we address the following questions, implicitly or explicitly raised in two recent publications [5], [22]:

1- if we employ the same theoretical/numerical model to simulate and invert the data (the inverse crime situation), are we sure to be able to retrieve the target solution?

2- how sensitive is this inverse crime solution (if it exists) to the choice of brackets of starting values of the to-be-retrieved parameters in the cost functional minimization scheme?

3- how sensitive is this inverse crime solution to parameters (e.g., number of function evaluations, number of iterations) inherent in the cost functional minimization scheme?

4- if we employ a retrieval model that differs from the data simulation model by the value of one of the priors, what is the best regularization strategy for generating a single solution to the inverse problem, and how close is this solution to the target solution?

5 - how sensitive is this non-inverse crime solution (if it exists) to the choice of range of starting values of the to-be-retrieved parameters? 


\section{Ingredients of multiparameter inversion of time- do- main data}

\subsection{The two models}

Any successful inversion of a data set (of physical origin) first requires a model of the physical process which accurately mimics the data [44]. We term this model, the retrieval model, or $\mathfrak{M}$. $\mathfrak{M}$ is characterized by its mathematical ingredients $\mathbf{M}$ and numerical ingredients $\mathbf{N}$.

When, as in the present study, the (true) data is not the result of a measurement, it must be generated (simulated), again with the help of a model of the underlying physical process which is thought to be able to mimic the true data. We term this model, the data simulation model, or $\mathfrak{m}$. $\mathfrak{m}$ is characterized by its mathematical ingredients $\mathbf{m}$ and numerical ingredient(s) $\mathbf{n}$.

Each model involves a certain number of physical/geometric parameters: those of $\mathfrak{m}$ and $\mathfrak{M}$ are $\mathbf{p}$ and $\mathbf{P}$ respectively, i.e., lower-case letters apply to the (true) data (simulated or experimental) and upper-case letters to the retrieval model.

\subsection{Basic retrieval scheme}

Let $\mathbf{r}:=\left\{r_{j} ; j=1,2, \ldots, N_{r}\right\}$ be the set of to-be-retrieved parameters, $\mathbf{q}:=\left\{q_{j} ; j=\right.$ $\left.1,2, \ldots N_{q}\right\}$ the set of priors (whose nature will be made more precise further on), $\mathbf{X}:=$ $\left\{\mathbf{x}_{j} ; j=1,2, . ., N_{x}\right\}$ the set of sensor locations and $\mathbf{t}:=\left\{t_{l} ; l=1,2, . ., N_{t}\right\}$ the set of instants of the sampled signal. Herein, $N_{r}=4$ and $N_{q}=9$.

The basic scheme is to replace the true (fixed) parameter set $\mathbf{r}$ by the trial (variable) parameter set $\mathbf{R}:=\left\{R_{1}, R_{2}, \ldots, R_{N_{r}}\right\}$ and the true (fixed) set of priors $\mathbf{q}$ by another fixed set $\mathbf{Q}:=\left\{Q_{1}, Q_{2}, \ldots, Q_{N_{q}}\right\}$, and search, in iterative manner, for the set $\mathbf{R}=\tilde{\mathbf{r}}$ that minimizes the discrepancy (the measure of which is a cost or objective functional) between trial signals (resulting from trial parameters) $\mathfrak{S}(\mathbf{X}, \mathbf{t}, \mathbf{Q}, \mathbf{R})$ and the true signal $\mathfrak{s}(\mathbf{X}, \mathbf{t}, \mathbf{q}, \mathbf{r})$ (resulting from the true parameters).

Note that if, as will be assumed in some of the examples presented hereafter, there exists some uncertainty in the priors, $\mathbf{Q}$ will not be identical to $\mathbf{q}$. In fact, this possible discordance will affect the precision of the retrieval and it is of considerable interest to quantify this effect.

\subsection{The inverse crime}

The inverse crime is something one should be concerned with when the two models $\mathfrak{m}$ and $\mathfrak{M}$ are identical (however, the values of the parameters $\mathbf{R}$ will differ from those of $\mathbf{r}$ during the inversion).

If a single parameter is to be retrieved, the condition for, and result of, committing the inverse crime can be stated [44] as follows: when the values of all the parameters except $P_{J}$ of the set $\mathbf{P}$ are strictly equal to their counterparts in the set $\mathbf{p}$ and the values of all 
the parameters of the set $\mathbf{N}$ are strictly equal to their counterparts in the set $\mathbf{n}$, then the inversion will give rise to at least one solution, $P_{J}=p_{J}$, i.e., the true solution.

If, as in the present case, more-than-one parameter is to be retrieved at one time, then it can be conjectured that the condition for, and result of, committing the inverse crime are: when the values of all the priors forming the subset $\mathbf{Q}$ of the set $\mathbf{P}$ are strictly equal to their counterparts in the subset $\mathbf{q}$ of the set $\mathbf{p}$, and the values of all the parameters of the set $\mathbf{N}$ are strictly equal to their counterparts in the set $\mathbf{n}$, then the inversion will give rise to at least one solution, $\mathbf{R}=\mathbf{r}$, i.e., the true solution.

This statement is only a conjecture because inverting for more than one parameter at a time usually requires the intervention of an optimization algorithm that may get stuck, or just appear to get stuck, in a local minimum of the objective (cost) functional (see further on how we define this functional), thereby giving rise to what appears to be a solution, which, in fact, is not the true solution $\mathbf{r}$ of the parameter retrieval problem. We shall give examples of this type of situation and of how it can be dealt with.

The eventuality of committing the inverse crime is highly improbable in real-life, in that the data is usually acquired physically (i.e., is not simulated), or the data is simulated but one has only a vague idea a priori of the value of at least one of the parameters of the sets $\mathbf{r}$ and/or q. This is the reason why, in the present study, we take explicitly in account these uncertainties, with the added benefit of being able to avoid the inverse crime.

\subsection{Minimization of the cost functional}

The discrepancy between $\mathfrak{S}$ and $\mathfrak{s}$ is the cost functional $\mathcal{K}$. We choose this as:

$$
\mathcal{K}(\mathbf{r}, \mathbf{R}, \mathbf{q}, \mathbf{Q}, \mathbf{X}, \mathbf{t})=\frac{\sum_{j=1}^{N_{x}} \sum_{k=1}^{N_{t}}\left[\mathfrak{s}\left(\mathbf{x}_{j}, t_{k}, \mathbf{q}, \mathbf{r}\right)-\mathfrak{S}\left(\mathbf{x}_{j}, t_{k}, \mathbf{Q}, \mathbf{R}\right)\right]^{2}}{\sum_{j=1}^{N_{x}} \sum_{k=1}^{N_{t}}\left[\mathfrak{s}\left(\mathbf{x}_{j}, t_{k}, \mathbf{q}, \mathbf{r}\right)\right]^{2} d t}
$$

The inverse problem is solved herein, at each stage, by minimization of the cost functional via the Nelder-Mead Simplex scheme [30]. The minimum of the cost functional (in the parameter space explored by the Simplex scheme, anchored at the user-defined starting values of the to-be-retrieved parameters, is found for $\mathbf{R}=\tilde{\mathbf{r}}$, i.e.,

$$
\tilde{\mathbf{r}}=\arg \min _{\mathbf{R} \subset \mathcal{S}} \mathcal{K}(\mathbf{r}, \mathbf{R}, \mathbf{q}, \mathbf{Q}, \mathbf{X}, \mathbf{t}),
$$

wherein $\mathcal{S}$ designates the parameter search space during a given stage of the search of a minimum, a stage being defined as a specific choice of the initial value set (since $f$ minsearch requests such a set to get started). We call the initial value set $\mathbf{R}:=\left\{R_{j} ; j=1,2,3, N_{r}=\right.$ $4\}$. Note that $\mathbf{R}$ takes on different values (from this initial value) as the minimization proceeds.

\subsection{The Simplex algorithm}

The simplex algorithm [26] is a geometrical rather than gradient-based optimization manner of simultaneously finding the $N_{r}$ parameters corresponding to the minimum of a (cost) 
function.

Few theoretical results have been proved explicitly concerning this algorithm, and then essentially in 1 and 2D dimensions [20]. Moreover, the method can fail to converge or converge to non-stationary solutions in certain classes of problems [23], [7]. Nevertheless, the Simplex algorithm has been frequently employed, particularly in parameter estimation problems [10], [18], [36], [5], [47], and even in problems involving noisy data [42], [40].

Although Byatt et al. [7] have reported on a supposedly-improved version (less function calls for a given value of the minimum of the cost function), we preferred herein to employ the off-the-shelf standard Simplex algorithm embodied in the MATLAB function $f$ minsearch.

Like other optimization schemes, the Simplex algorithm is iterative by nature, with each iteration requiring a certain number of (cost) function computations. The number of iterations and function computations increases with: a) the precision with which one wants to locate the minimum of the (cost) function, b) the distance of the initial values of the parameters to their target values, and c) the topological nature of the cost function (see [7] for an illustration of this fact).

In $f$ minsearch, the maximum number of function evaluations and maximum number of iterations can be chosen, via the parameters $M F E$ and $M I$ respectively, by the user. This appears to us to be a better strategy than choosing the precision, because aiming at a high precision may require a prohibitive amount of function evaluations and/or iterations.

\subsection{Parameter retrieval schemes}

The schemes considered herein actually solve for all members of the set $\mathbf{r}$ at each stage.

Often, a priori information, concerning the solutions of the inverse problem, is of the form: 'the sought-for parameter $r_{j}$ lies between $R_{b j}$ and $R_{e j}$ ', which fact suggests that one should try to carry out the retrieval for a variety of starting values lying between $R_{b j}$ and $R_{e j}$.

More precisely, we shall carry out the inversion for the set of starting values $\mathbf{R}:=$ $\left\{R_{j k} ; j=1,2,3, N_{r}=4 ; k=1,2, \ldots, N_{R_{j}}\right\}$, with

$$
R_{j k}:=R_{b j}+(k-1) d_{R_{j}} ; j=1,2,3,4 ; k=1,2, \ldots, N_{R_{j}},
$$

and

$$
d_{R_{j}}:=\frac{R_{e j}-R_{b j}}{N_{R_{j}}-1}
$$

Thus, supposing $N_{R_{1}}=N_{R_{2}}=N_{R_{3}}=N_{R_{4}}$ := $N_{R}$, the number of stages, and the number of retrieved solutions, will be $N_{R}^{4}$, a number that increases rapidly with $N_{R}$ since $2^{4}=16,31$ $3^{4}=81,4^{4}=256,5^{4}=625, \ldots$

The question is then how to select the most appropriate (i.e., 'best') solution among this multitude of retrievals.

Let $\tilde{r}_{j k}$ designate the $j$-th retrieved parameter at the $k$-th stage and let $\min \mathcal{K}_{k}$ be the value of the minimum of the cost function found by the Simplex scheme at the $k$-th stage. 
A zeroth-order (so-called min min cost) regularization proceeds as follows: the final retrieved value of $r_{j}$ (after completing all the $N_{R_{j}}$ stages) is designated by $\tilde{r}_{j}$ and is chosen amongst all the $\tilde{r}_{j k}$, to be the one corresponding to the minimum of the set $\left\{\min \mathcal{K}_{k} ; k=\right.$ $1,2, \ldots, N\}$.

A first-order regularization consists in rejecting solutions for which one or more retrieved parameters are not physical (e.g., a positve quantity that turns out to be negative) and then applying the min min cost rule to the remaining solutions.

The result of an inversion scheme can lead to (one or more) $r_{j}$ that are not within the initial search interval $\left[R_{b j}, R_{e j}\right]$, but a logical option (i.e., the second-order regularization scheme) is to reject such retrievals (otherwise, why impose initial search intervals other than to constrain the retrievals?) and then apply the min min cost rule to the remaining solutions.

In the sequel, we shall apply, and attempt to evaluate, these regularization schemes to our canonical inverse problem.

\subsection{The retrieval error generated by prior uncertainty}

Nonlinear inversion usually leads to error-ridden retrievals of the $j$-th parameter (recall that the true value of this parameter is $r_{j}$ ). Our aim herein will be to evaluate the relative error of the final retrieved parameter $\tilde{r}_{j}\left(j=1,2, \ldots, N_{r}=4\right)$

$$
\varepsilon_{j}:=\frac{\tilde{r}_{j}-r_{j}}{r_{j}}
$$

as a function of the prior uncertainties, which, for the $k$-th prior, is

$$
\delta_{k}:=\frac{Q_{k}-q_{k}}{q_{k}}
$$

We shall also evaluate the cumulative relative error of the retrieved parameters, defined as

$$
\kappa=\sum_{j=1}^{N_{r}}\left|\varepsilon_{j}\right| .
$$

\section{The models for computing the signals $\mathfrak{s}\left(\mathbf{x}_{j}, t_{k}, \mathbf{q}, \mathbf{r}\right)$ and $\mathfrak{S}\left(\mathbf{x}_{j}, t_{k}, \mathbf{Q}, \mathbf{R}\right)$}

\subsection{Model description}

An infinitely-long (in the $z$-direction of an $O x y z$ cartesian reference system) lossy fluid-like cylinder, (known to be) centered at the origin $O$ and of radius $a$, is immersed in another (host, non-lossy) fluid and is submitted to an acoustic plane wave whose wavevector $\mathbf{k}^{i}$ lies in the $x-y$ (sagittal) plane. Consequently, the incident and diffracted fields are independent of $z$, i.e., the problem is $2 \mathrm{D}$, with $z$ the ignorable ooordinate. 
The plane wave carries a pulse, and the purpose of this excitation field is to produce a pulse-like diffracted field constituting the data, which by means of an inversion scheme, is analyzed to enable the retrieval of the radius of the cylinder and constitutive parameters of the fluid enclosed therein.

The constitutive parameters of the host medium, as well as the excitation parameters, may, or may not, be precisely known beforehand.

The analysis takes place in the sagittal plane wherein $\Omega_{0}$ denotes the domain exterior to the cylinder, $\Gamma$ the circular bounding curve of the cylinder, and $\Omega_{1}$ the domain within $\Gamma$.

The homogeneous fluids in $\Omega_{l}$ are $M^{[l]}, l=0,1$. The acoustic bulk phase velocities in these media are $c^{[l]}=\Re c^{[l]}+\Im c^{[l]}$, with $\Im c^{[1]} \leq 0$ and $\Im c^{[0]}=0$. The densities in $M^{[l]}$, $l=0,1$ are $\rho^{[l]}$. The corresponding wavenumbers are $k^{[l]}:=\omega / c^{[l]}$, with $\omega=2 \pi f$ the angular frequency and $f$ the frequency.

The bandwidth of the incident pulse is, for all practical purposes, finite, and within this band the phase velocities of the two fluids are assumed to be constant with respect to the frequency $f$.

The (incident) angle between $\mathbf{k}^{i}$ and the $x$ axis is $\theta^{i}$. This angle may, or may not, be precisely known.

The diffracted field is sensed at various $\left(N_{x}\right)$ points $\mathbf{X}$ on a circle of radius $b>a$ enclosing the cylinder and having the common origin $O$. The polar angle at which a generic point-like sensor is located is $\theta_{j}$ (with respect to the positive $x$ axis). $\mathbf{X}$ is assumed to be precisely known.

The objective is to retrieve the four parameters $c^{[1] r}:=\Re c^{[1]}, c^{[1] i}:=\Im c^{[1]}, \rho^{[1]}$ and a from data relating to the diffracted (scattered) field signals recorded at one or more sensors.

The term "diffracted field signals recorded..." is actually improper. In reality, two recordings are carried out: the first in the absence of the cylinder so as to obtain the incident field signal at the various sensors, and the second in the presence of the cylinder so as to obtain the total field signal at the various sensors, with the diffracted field signal at a given sensor being the total field signal minus the incident field signal.

Recall that in this study, the experiment for obtaining the diffracted field data signal(s), rather than being carried out physically in the laboratory or field, is simulated by computational means via the data simulation model, whereas the inversion requires another model, termed the retrieval model. We assume the mathematical and numerical ingredients of these two models to be identical; the two models differ only by the values of various physical/geometrical parameters entering therein.

Each model is employed to solve a direct scattering problem, once as concerns the generation of the synthetic data, and as many times as necessary during the inversion process.

\subsection{Acoustic field solutions}

The total frequency-domain pressure fields are:

$$
u^{[0]}(\mathbf{x}, \omega)=u^{[0] i}(\mathbf{x}, \omega)+u^{[0] d}(\mathbf{x}, \omega) ; \mathbf{x} \in \Omega_{0},
$$




$$
u^{[1]}(\mathbf{x}, \omega)=u^{[1] d}(\mathbf{x}, \omega) ; \mathbf{x} \in \Omega_{1} .
$$

with $\mathbf{x}$ denoting a vector joining $O$ to a generic point in the sagittal plane and $r, \theta$ the polar coordinates of this generic point.

The incident plane-wave field is:

$$
u^{[0] i}(\mathbf{x}, \omega)=S(\omega) e^{-i k r \cos \left(\theta-\theta^{i}\right)}=S(\omega) \sum_{m \in \mathbb{Z}} \gamma_{m} J_{m}\left(k^{[0]} r\right) e^{i m \theta},
$$

wherein $S(\omega)$ (such that $S(-\omega)=S^{*}(\omega), *$ designating the complex conjugate) is the spectrum of the pulse associated with the incident wave, and

$$
\gamma_{m}=e^{-i m\left(\theta^{i}+\frac{\pi}{2}\right)} .
$$

We choose a pseudo-Ricker type of pulse excitation whose spectrum is

$$
S(\omega)=\frac{\omega^{2}}{4 \alpha^{3} \sqrt{\pi}} \exp \left(i \beta \omega-\frac{\omega^{2}}{4 \alpha^{2}}\right) .
$$

The diffracted fields are (Wirgin, 1999):

$$
\begin{aligned}
& u^{[0] d}(\mathbf{x}, \omega)=S(\omega) \sum_{m \in \mathbb{Z}} \mathfrak{A}_{m} H_{m}^{(1)}\left(k^{[0]} r\right) e^{i m \theta} \\
& u^{[1] d}(\mathbf{x}, \omega)=S(\omega) \sum_{m \in \mathbb{Z}} \mathfrak{B}_{m} J_{m}\left(k^{[1]} r\right) e^{i m \theta} .
\end{aligned}
$$

wherein

$$
\begin{gathered}
\mathfrak{A}_{m}=\gamma_{m} A_{m}, \\
A_{m}=\frac{J_{m}\left(k^{[0]} a\right) \dot{J}_{m}\left(k^{[1]} a\right)-\eta \dot{J}_{m}\left(k^{[0]} a\right) J_{m}\left(k^{[1]} a\right)}{\eta \dot{H}_{m}^{(1)}\left(k^{[0]} a\right) J_{m}\left(k^{[1]} a\right)-H_{m}^{(1)}\left(k^{[0]} a\right) \dot{J}_{m}\left(k^{[1]} a\right)}, \\
\eta:=\frac{k^{[0]} \rho^{[1]}}{k^{[1]} \rho^{[0]}}, \\
\dot{Z}_{m}(z):=\frac{d}{d z} Z_{m}(z)=-Z_{m+1}(z)+\frac{m}{z} Z_{m}(z),
\end{gathered}
$$

with $Z_{m}(z)=J_{m}(z)$ (Bessel function) or $Z_{m}(z)=H_{m}^{(1)}(z)$ (Hankel function).

Due to the fact that $\gamma_{-m}=\gamma_{m}^{*}$ and

$$
Z_{-m}(z)=(-1)^{m} Z_{m}(z),
$$

we find that $A_{-m}=A_{m}$, whence

$$
u^{[0] d}(\mathbf{x}, \omega)=\sum_{m=0}^{\infty} A_{m} \epsilon_{m}(-i)^{m} H_{m}^{(1)}\left(k^{[0]} r\right) \cos \left(m\left(\theta-\theta^{i}\right)\right),
$$


with $\epsilon_{0}=1, \epsilon_{m>0}=2$.

The time-domain diffracted field outside the cylinder is

$$
U^{[0] d}(\mathbf{x}, t)=\int_{-\infty}^{\infty} u^{[0] d}(\mathbf{x}, \omega) e^{-i \omega t} d \omega
$$

and since (as can be shown) $u^{[0] d}(\mathbf{x},-\omega)=\left(u^{[0] d}(\mathbf{x}, \omega)\right)^{*}$, it follows that

$$
U^{[0] d}(\mathbf{x}, t)=2 \Re \int_{0}^{\infty} u^{[0] d}(\mathbf{x}, \omega) e^{-i \omega t} d \omega
$$

\section{Numerical issues}

We truncate the series in $u^{[0] d}(\mathbf{x}, \omega)$ in the following manner

$$
u^{[0] d}(\mathbf{x}, \omega) \approx u^{[0] d N_{f}}(\mathbf{x}, \omega)=\sum_{m=0}^{N_{f}} A_{m} \epsilon_{m}(-i)^{m} H_{m}^{(1)}\left(k^{[0]} r\right) \cos \left(m\left(\theta-\theta^{i}\right)\right)
$$

wherein

$$
N_{f}=\mathfrak{f} k^{[0]} b+3,
$$

with $\mathfrak{f}$ a user-chosen factor that is usually equal to 2 or 3 .

We carry out the Fourier transform as follows

$$
U^{[0] d}(\mathbf{x}, t) \approx 2 \Re \int_{2 \pi f_{d}}^{2 \pi f_{f}} u^{[0] d N_{f}}(\mathbf{x}, \omega) e^{-i \omega t} d \omega
$$

with $f_{d}$ a very small number, $f_{f}$ chosen large enough to prevent aliasing, and the finite integral evaluated by the Simpson scheme. 


\section{The parameters involved in the simulated data and retrieval data}

\subsection{The parameters involved in the (simulated) data}

Our simulated data involves fourteen physical/geometric parameters (PGP) and two numerical parameter (NP) sets, both designated by lower-case characters. The PGP consist

\begin{tabular}{|c|c|c|c|c|c|c|c|c|c|c|c|c|c|}
\hline$p_{1}$ & $p_{2}$ & $p_{3}$ & $p_{4}$ & $p_{5}$ & $p_{6}$ & $p_{7}$ & $p_{8}$ & $p_{9}$ & $p_{10}$ & $p_{11}$ & $p_{12}$ & $p_{13}$ & $p_{14}$ \\
\hline$r_{1}$ & $r_{2}$ & $r_{3}$ & $r_{4}$ & $q_{1}$ & $q_{2}$ & $q_{3}$ & $q_{4}$ & $q_{5}$ & $q_{6}$ & $q_{7}$ & $q_{8}$ & $q_{9}$ & $q_{10}$ \\
\hline$c^{[1] r}$ & $c^{[1] i}$ & $\rho^{[1]}$ & $a$ & $c^{[0]}$ & $\rho^{[0]}$ & $\theta^{i}$ & $\alpha$ & $\beta$ & $b$ & $\theta_{b}$ & $\theta_{e}$ & $N_{\theta}$ & $\mathcal{P}$ \\
\hline
\end{tabular}

Table 1: Correspondences between the different (lower case) symbols relating to the simulated/experimental (fixed during the retrieval) parameters. The entries of a given column represent the same parameter.

of: the four to-be-retrieved parameters of the cylinder $p_{1}, p_{2}, p_{3}, p_{4}$, the two background medium priors $p_{5}, p_{6}$, the three excitation priors $p_{7}, p_{8}, p_{9}$ and the five receiver priors $p_{10}, p_{11}, p_{12}, p_{13}, p_{14}$.

The polar angle of the $j$-th receiver (the total number of receivers being $N_{\theta}$ ) is

$$
\theta_{j}=\theta_{b}+(j-1) d_{\theta},
$$

and

$$
d_{\theta}=\frac{\theta_{e}-\theta_{b}}{N_{\theta}-1}
$$

it being understood that

$$
\mathfrak{s}\left(\mathbf{x}_{j}, t_{k}, \mathbf{q}, \mathbf{r}\right)=U^{[0] d}\left(b, \theta_{j}, t_{k}, \mathbf{q}, \mathbf{r}\right)
$$

is the (simulated) data signal at the $j$-th receiver and instant $t_{k}$, and that

$$
\mathfrak{S}\left(\mathbf{x}_{j}, t_{k}, \mathbf{Q}, \mathbf{R}\right)=U^{[0] d}\left(b, \theta_{j}, t_{k}, \mathbf{Q}, \mathbf{R}\right),
$$

is the retrieval signal at the same receiver and instant.

In practice, the retrieved signal is affected by noise whose amplitude is accounted-for by the parameter $\mathcal{P}$, but in the sequel we consider only noiseless data so that $\mathcal{P}=0$.

The two NP sets are: $\mathbf{n}_{1}=\left(f_{d}, f_{f}, N_{f}, \mathfrak{f}\right), \mathbf{n}_{2}=\left(t_{d}, t_{f}, N_{t}\right)$. We stress the fact that the first two NP employed in the retrieval model are identical to those in the data simulation model, i.e., $\mathbf{N}_{l}=\mathbf{n}_{l} ; l=1,2$. 


\subsection{The parameters involved in the retrieval data}

Our retrieval data involves fourteen physical/geometric (PGP) parameter sets and two numerical parameter (NP) sets, both designated by upper-case characters.

\begin{tabular}{|c|c|c|c|c|c|c|c|c|c|c|c|c|c|}
\hline $\mathbf{P}_{1}$ & $\mathbf{P}_{2}$ & $\mathbf{P}_{3}$ & $\mathbf{P}_{4}$ & $P_{5}$ & $P_{6}$ & $P_{7}$ & $P_{8}$ & $P_{9}$ & $P_{10}$ & $P_{11}$ & $P_{12}$ & $P_{13}$ & $P_{14}$ \\
\hline $\mathbf{R}_{1}$ & $\mathbf{R}_{2}$ & $\mathbf{R}_{3}$ & $\mathbf{R}_{4}$ & $Q_{1}$ & $Q_{2}$ & $Q_{3}$ & $Q_{4}$ & $Q_{5}$ & $Q_{6}$ & $Q_{7}$ & $Q_{8}$ & $Q_{9}$ & $Q_{10}$ \\
\hline $\begin{array}{c}\left(C_{b}^{[1] r},\right. \\
C_{e}^{[1] r}, \\
\left.N_{C}^{[1] r}\right)\end{array}$ & $\begin{array}{c}C_{b}^{[1] i}, \\
C_{e}^{[1] i}, \\
N_{C}^{[1] i}\end{array}$ & $\begin{array}{c}\left(\mathcal{R}_{b}^{[1]},\right. \\
\mathcal{R}_{e}^{[1]}, \\
\left.N_{\mathcal{R}^{[1]}}\right)\end{array}$ & $\begin{array}{c}\left(A_{b},\right. \\
A_{e}, \\
N_{A}\end{array}$ & $C^{[0]}$ & $\mathcal{R}^{[0]}$ & $\Theta^{i}$ & $\mathcal{A}$ & $\mathcal{B}$ & $B$ & $\Theta_{b}$ & $\Theta_{e}$ & $N_{\Theta}$ & $\mathcal{P}$ \\
\hline
\end{tabular}

Table 2: Correspondences between the different (upper case) symbols relating to the retrieval parameters. The entries of a given column represent the same parameter or vector.

The PGP consist of: the four parameter sets relative to the cylinder $\mathbf{P}_{1}, \mathbf{P}_{2}, \mathbf{P}_{3}, \mathbf{P}_{4}$, the two background priors $P_{5}, P_{6}$, the three excitation priors $P_{7}, P_{8} P_{9}$, and the five receiver priors $P_{10}, P_{11} P_{12}, P_{13}, P_{14}$, it being understood that

$$
\mathbf{P}_{j}=\mathbf{R}_{j}=\left(R_{b j}, R_{e j}, N_{R_{j}}\right) ; j=1,2,3,4
$$

and that we choose: $P_{j}=p_{j} ; j=10,12,13,14$.

Furthermore,

$$
\mathfrak{S}\left(\mathbf{x}_{j}, t_{k}, \mathbf{Q}, \mathbf{R}\right)=U^{[0] d}\left(b, \theta_{j}, t_{k}, \mathbf{Q}, \mathbf{R}\right)
$$

is the retrieval signal at the $j$-th receiver and instant $t_{k}$.

The three numerical parameter sets are: $\mathbf{N}_{1}=\left(F_{d}, F_{f}, N_{F}, \mathfrak{F}\right), \mathbf{n}_{2}=\left(t_{d}, t_{f}, N_{t}\right), \mathbf{n}_{3}=$ $(M F E)$, wherein $M F E$ is the maximum number of function evaluations per stage in each call to the Simplex function.

We always chose $\mathbf{N}_{l}=\mathbf{n}_{l} ; l=1,2$. 


\section{Numerical results for the inverse crime situation}

\subsection{Parameters and conventions common to the figures}

The true (target) parameters are: $c^{[1] r}=1700 \mathrm{~m} / \mathrm{s}, c^{[1] i}=-210 \mathrm{~m} / \mathrm{s}, \rho^{[1]}=1300 \mathrm{~kg} / \mathrm{m}^{3}$, $a=.1 \mathrm{~m}$.

The priors are as follows: $C^{[0]}=c^{[0]}=1500 \mathrm{~m} / \mathrm{s}, \quad \rho^{[0]}=R^{[0]}=\rho^{[0]}=1000 \mathrm{~kg} / \mathrm{m}^{3}, \quad \Theta^{i}=$ $\theta^{i}=0^{\circ}, \quad \mathcal{A}=\alpha=2000 \mathrm{~Hz}, \quad \mathcal{B}=\beta=.1 \mathrm{~s}, \quad B=b=1 \mathrm{~m}$.

The numerical parameters are: $\mathbf{f}=(.003 \mathrm{~Hz}, 2000 \mathrm{~Hz}, 201), \quad \mathbf{t}=(.098 \mathrm{~s}, .103 \mathrm{~s}, 181)$. Unless specified otherwise, we take $\boldsymbol{\theta}=\left(0^{\circ}, 180^{\circ}, 5\right)$.

As stated earlier, we do possess some knowledge at the outset of the values of the parameters we wish to retrieve. This knowledge takes the form of intervals which are used to initialize the Simplex optimization algorithm.

To parametrize this degree of knowledge, we employ the variable $d_{R_{j}}=d ; j=1,2,3,4$, which gives a measure of the width of the initial parameter intervals $\left[C_{b}^{[1] r}=c^{[1] r}(1-\right.$ $\left.d), C_{e}^{[1] r}=c^{[1] r}(1+d)\right],\left[C_{b}^{[1] i}=c^{[1] i}(1-d), C_{e}^{[1] i}=c^{[1] i}(1+d)\right],\left[\mathcal{R}_{b}^{[1]}=\rho^{[1]}(1-d), \mathcal{R}_{e}^{[1]}=\right.$ $\left.\rho^{[1]}(1+d)\right],\left[A_{b}=a(1-d), A_{e}=a(1+d)\right]$.

In all the numerical examples of this study, only the endpoints of these intervals will be used as initial values in the Simplex multistage scheme, so that each of the following figures will be the result of a $16\left(=2^{4}\right)$ stage computation (see table 3 ).

\begin{tabular}{|c|c|c|c|c|}
\hline$d$ & $\mathbf{R}_{1}$ & $\mathbf{R}_{2}$ & $\mathbf{R}_{3}$ & $\mathbf{R}_{4}$ \\
\hline .1 & $(1530,1870,2)$ & $(-231,-189,2)$ & $(1170,1430,2)$ & $(.09, .11,2)$ \\
.2 & $(1360,2040,2)$ & $(-252,-168,2)$ & $(1040,1560,2)$ & $(.08, .12,2)$ \\
.3 & $(1190,2210,2)$ & $(-273,-147,2)$ & $(910,1670,2)$ & $(.07, .13,2)$ \\
.4 & $(1020,2380,2)$ & $(-294,-126,2)$ & $(780,1820,2)$ & $(.06, .14,2)$ \\
.5 & $(850,2550,2)$ & $(-315,-105,2)$ & $(650,1950,2)$ & $(.05, .15,2)$ \\
.6 & $(680,2720,2)$ & $(-336,-84,2)$ & $(520,2080,2)$ & $(.04, .16,2)$ \\
.7 & $(510,2890,2)$ & $(-357,-63,2)$ & $(390,2210,2)$ & $(.03, .17,2)$ \\
.8 & $(340,3060,2)$ & $(-378,-42,2)$ & $(260,2340,2)$ & $(.02, .18,2)$ \\
.9 & $(170,3230,2)$ & $(-399,-21,2)$ & $(130,2470,2)$ & $(.01, .19,2)$ \\
& & & & .1 \\
\hline target & 1700 & -210 & 1300 & \\
\hline
\end{tabular}

Table 3: Correspondences between $d_{R_{j}}=d ; j=1,2,3,4$ and $\mathbf{R}_{j}$ in the initial search intervals. The last row indicates the target (true) values of the four to-be-retrieved parameters. The larger is d the more imprecise is the knowledge one has of the values of the sought-for parameters at the beginning of the retrieval procedure. Most of our numerical examples will be carried out for $d=.9$. 


\subsubsection{A rule for spotting admissible solutions}

Each of the following three figures (i.e., figs. 1-6), and many of the subsequent figures, contain four panels within which appear 16 points resulting from the multistage inversion procedure.

The abscissas of the graphs in each panel represent the value of the minimum of the cost function $\mathcal{K}_{k}$ found by the Simplex scheme at the $k$-th stage of the inversion procedure.

The ordinates of the graphs in these panels represent the four to-be-retrieved parameters: $c^{[1] r}$ (first panel from the left), $c^{[1] i}$ (second panel from the left), $\rho^{[1]}$ (third panel from the left), and $a$ (fourth panel from the left).

The horizontal (blue) segments designate the true values of these four parameters, so that the closer one of the 16 points is to this segment, the closer the retrieved parameter is to its true value.

Since, in practice, we do not know the true values of the sought-for parameters, we must take a decision as to which of these multiple solutions (each one corresponding to a point on the graphs) are admissible. Often, the solutions generated by the minimization scheme separate into two groups: to the first correspond rather small values of the minimum of the cost functional $\mathcal{K}$, and to the second correspond rather large (usually, at least one order of magnitude larger than the $\min \mathcal{K}$ of the first group) values of the minimum of $\mathcal{K}$.

It is the set of points furthest to the left in each panel which correspond to admissible solutions. Hopefully, the points associated with small $\min \mathcal{K}_{k}$ also correspond to those that are closest to the horizontal segments.

\subsection{A first result analogous to those of Buchanan et al. [5]}

\subsubsection{The Buchanan et al. procedure}

The publication of Buchanan et al. [5], although applying to a different canonical scattering body (their's is a poroelastic panel), is of interest here because:

1- the context is that of the inverse crime as is ours in the six figures which follow;

2- Buchanan et al. also deal with a multiparameter nonlinear full-wave inversion of time domain data,

3- their retrieval is done via the same Simplex algorithm as our's;

4- they adopt the same 16 stage procedure as our's to retrieve four constitutive parameters.

Their figs. 6-8 each show the combined result of three 16-stage inversions corresponding to choosing $d=.1, .2, .3$, so that 48 points are present in each figure. They write: "As no noise or modeling error was present, a nearly exact recovery was possible, but this happened in only one, sample M1F04, of the six attempts. Figures 6-8 illustrate the presence of numerous local minima of the objective function...". 


\subsubsection{Our procedure}

We separated the three cases $d=.1, .2, .3$ into three separate figures 1-3, but the result is qualitatively the same as that in [5] if we imagine our three figures to be superimposed onto a single figure. Our separation procedure has the advantage of showing that the result is clearly worse (in the sense of the previously-cited phrase of [5]) for larger initial parameter interval widths (as measured by d).
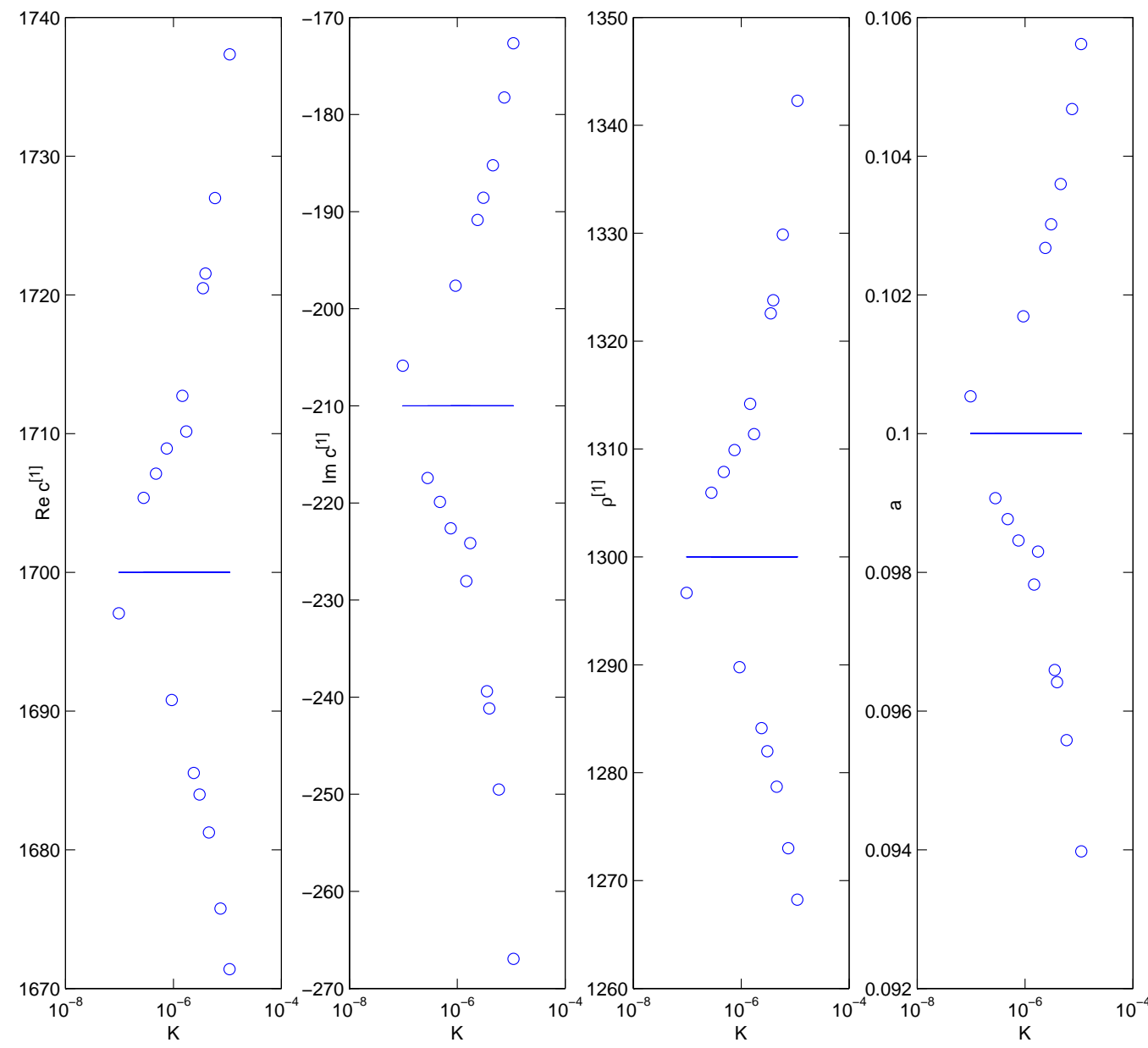

Figure 1: Case 270213-1529. $d=.1 . M F E=100$. 


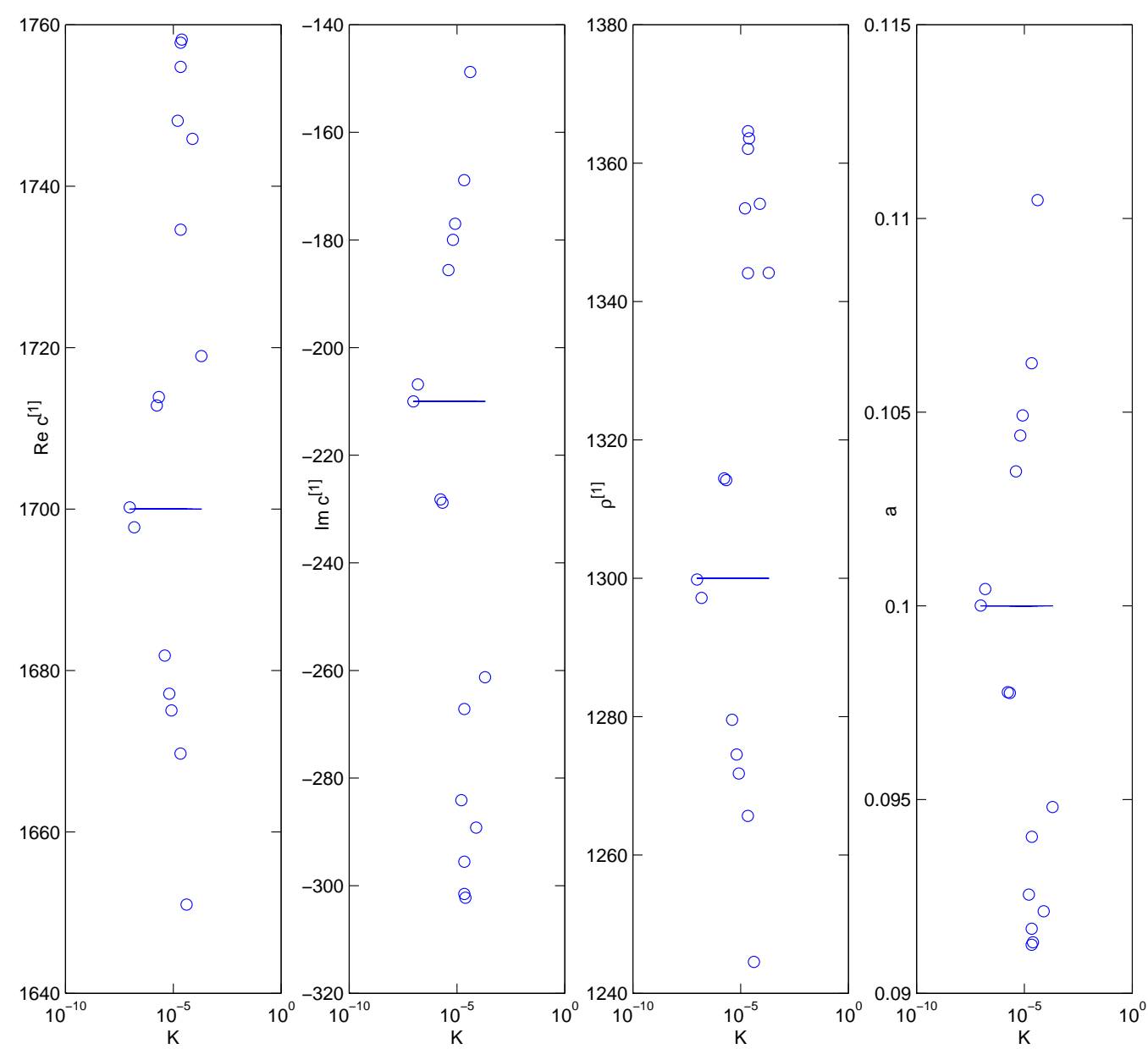

Figure 2: Case 270213-1529. $d=.2 . \quad M F E=100$. 


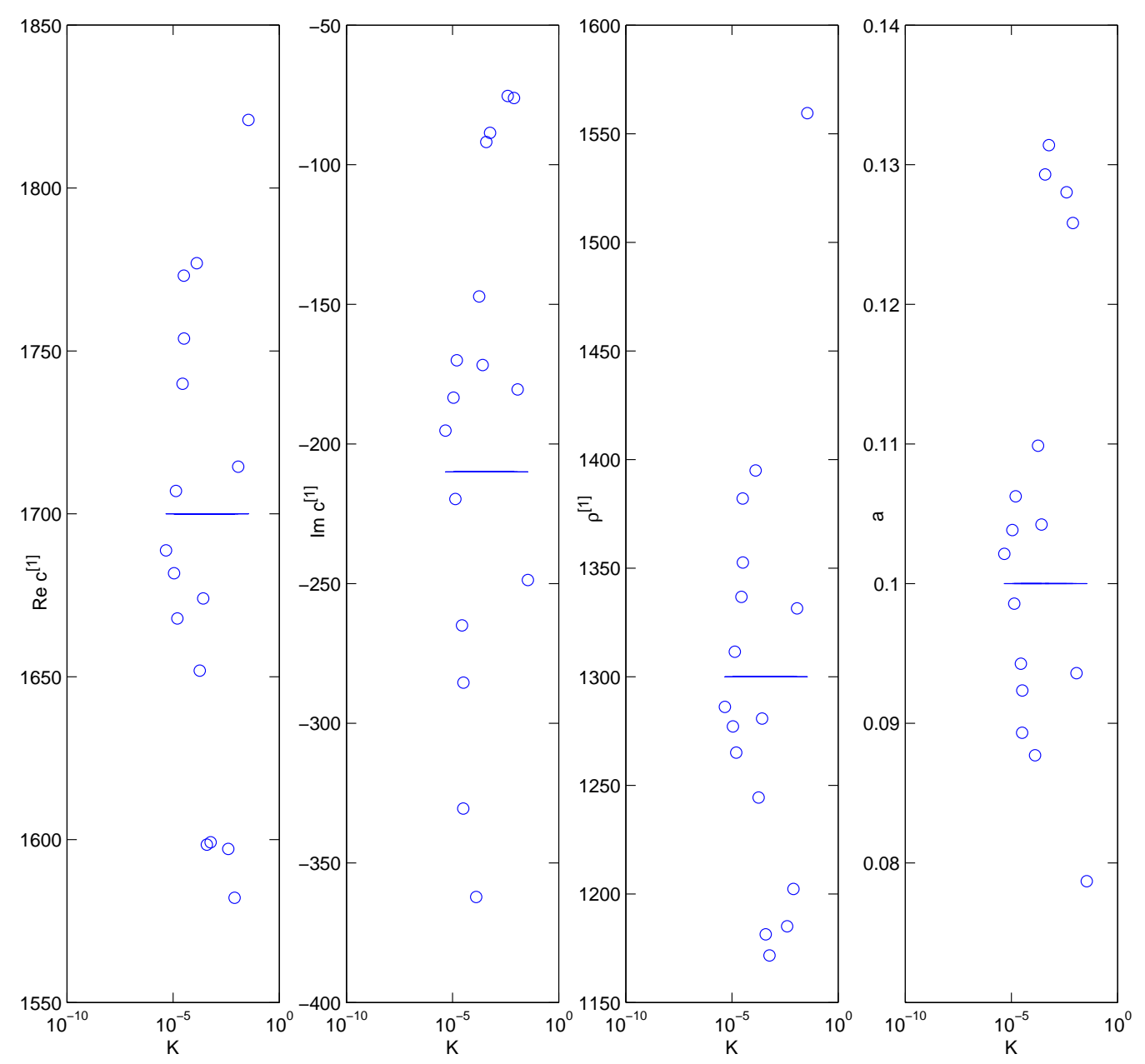

Figure 3: Case 270213-1529. $d=.3 . \quad M F E=100$. 


\subsubsection{Comments on the Buchanan et al. procedure}

It is not clear how Buchanan et al. tuned their Simplex routine, notably as concerns the choice of the parameter $M F E$ (maximum function evaluations) (and perhaps $M I$ (maximum number of iterations)), but it may be that $M F E$ was relatively-small (as in our figs. 1-3).

The Simplex algorithm actually exits whenever the function evaluation counter (and/or the iteration counter, if the $M I$ is specified) attains $M F E$ (and/or $M I$ ), and if $M F E$ is too small, the algorithm yields a 'solution' (a set of 16 points) to which corresponds a value of the cost function that can be far from its actual minimum. Thus, it may be, that these points, rather than those corresponding to local minima, are simply false solutions due to the inversion procedure having being stopped too early.

\subsection{Subsequent results that go beyond the one analogous to those of Buchanan, 2011}

This hypothesis drove us to increase $M F E$, thereby leading to the results in figs. 4-6 wherein we observe that the distribution of points is no longer haphazard. In fact, most of the points seem to converge to a single location which happens to be the target value for which the cost function is minimal (and in fact very small).

This implies that if we let the Simplex scheme do its work to completion (actually this could go on even longer, and the tendency of convergence be accentuated, until machine roundoff errors destroy the result), it will generate a single solution which happens to coincide with the exact solution. This finding seems to be true whatever the initial parameter interval width, but the convergence rate is observed to be slower, and the computation requires larger $M F E$, the larger the initial parameter interval width. 

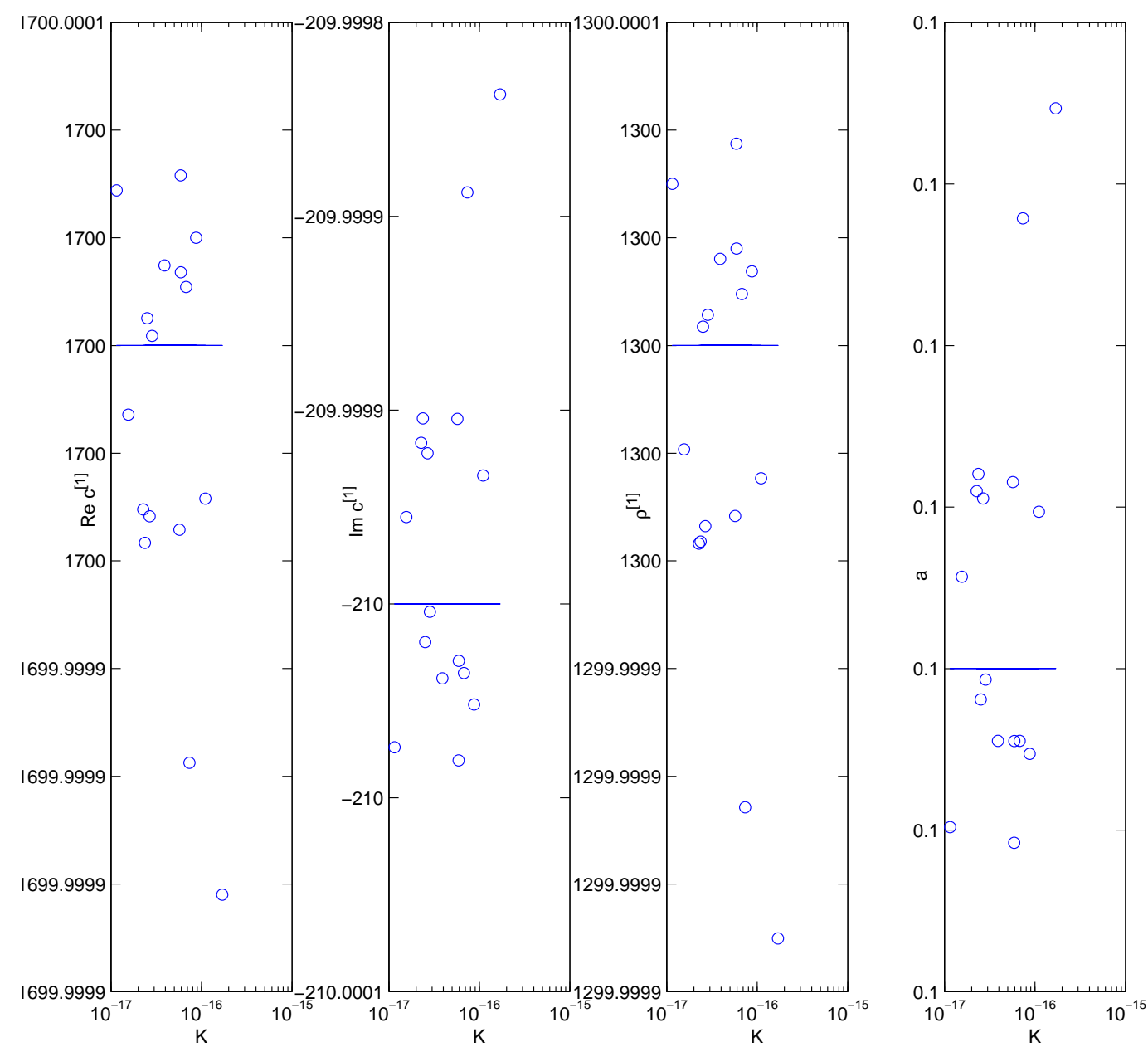

Figure 4: Case 270213-1554. $d=.1 . M F E=500$. Note the greatly magnified scale, so that this cloud of points appears as a single point at the scale of the next two figures. 

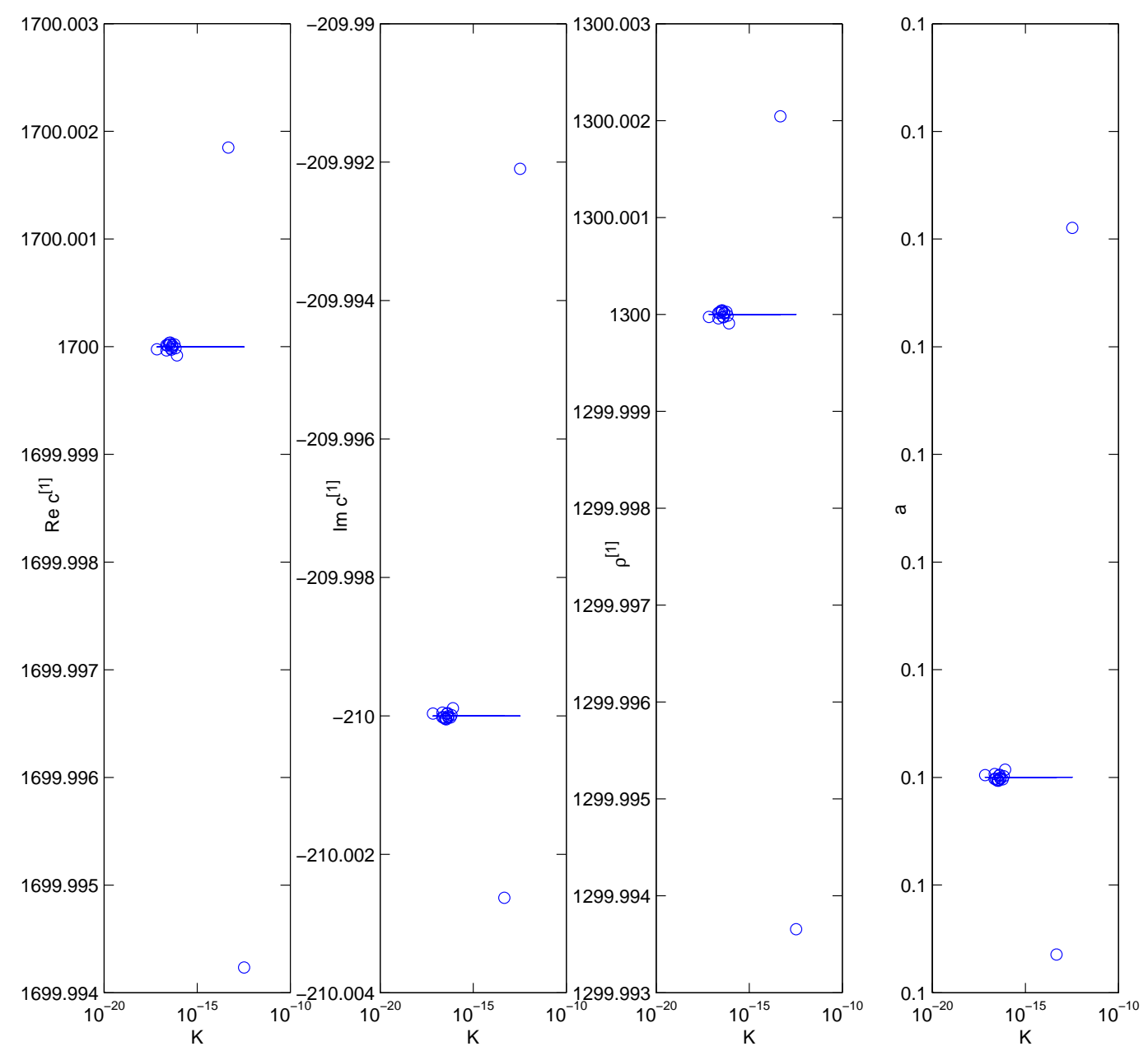

Figure 5: Case 270213-1554. $d=.2 . \quad M F E=500$. 

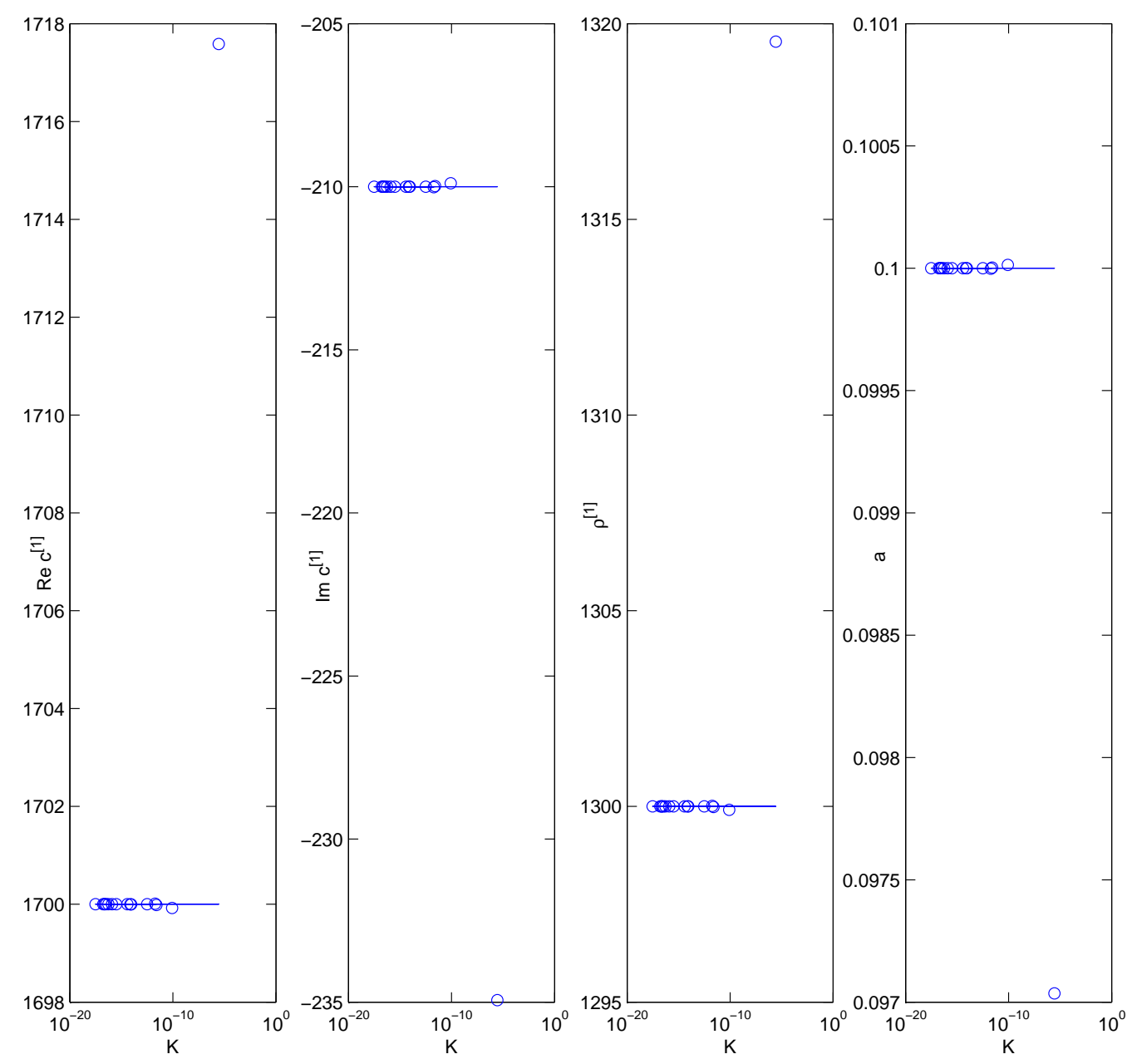

Figure 6: Case 270213-1554. $d=.3 . M F E=500$. 


\subsection{Effect of the variation of $M F E$ for a large initial parameter interval $d_{R_{j}}=d=.9 ; j=1,2,3,4$.}

The results of the previous section show that the choice of the Simplex parameter MFE determines to a large extent the success of the parameter retrieval, all the more so the larger is the initial parameter intervals. This was found for relatively-small initial parameter intervals (i.e., $d=.1, .2, .3)$ to conform to what was done in Buchanan et al., 2011. At present, we want to determine whether it is possible to retrieve what looks like a unique solution, and how close the latter is to the target, when the initial parameter interval is large.

The following set of figures corresponds to increasing values of $M F E$ and is again presented in the form borrowed from Buchanan et al., 2011. 


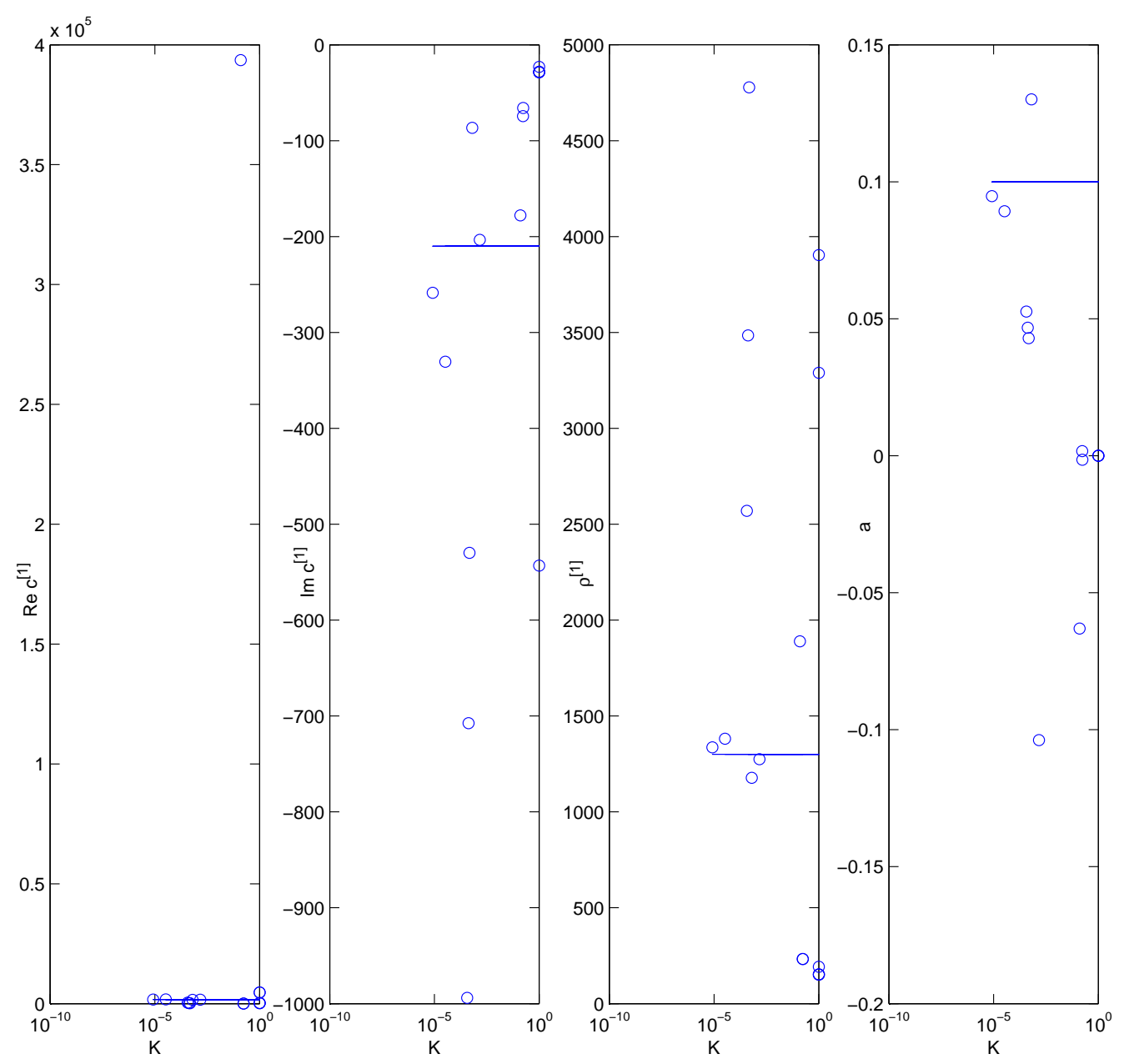

Figure 7: Case 250213-0726. $M F E=500$. 

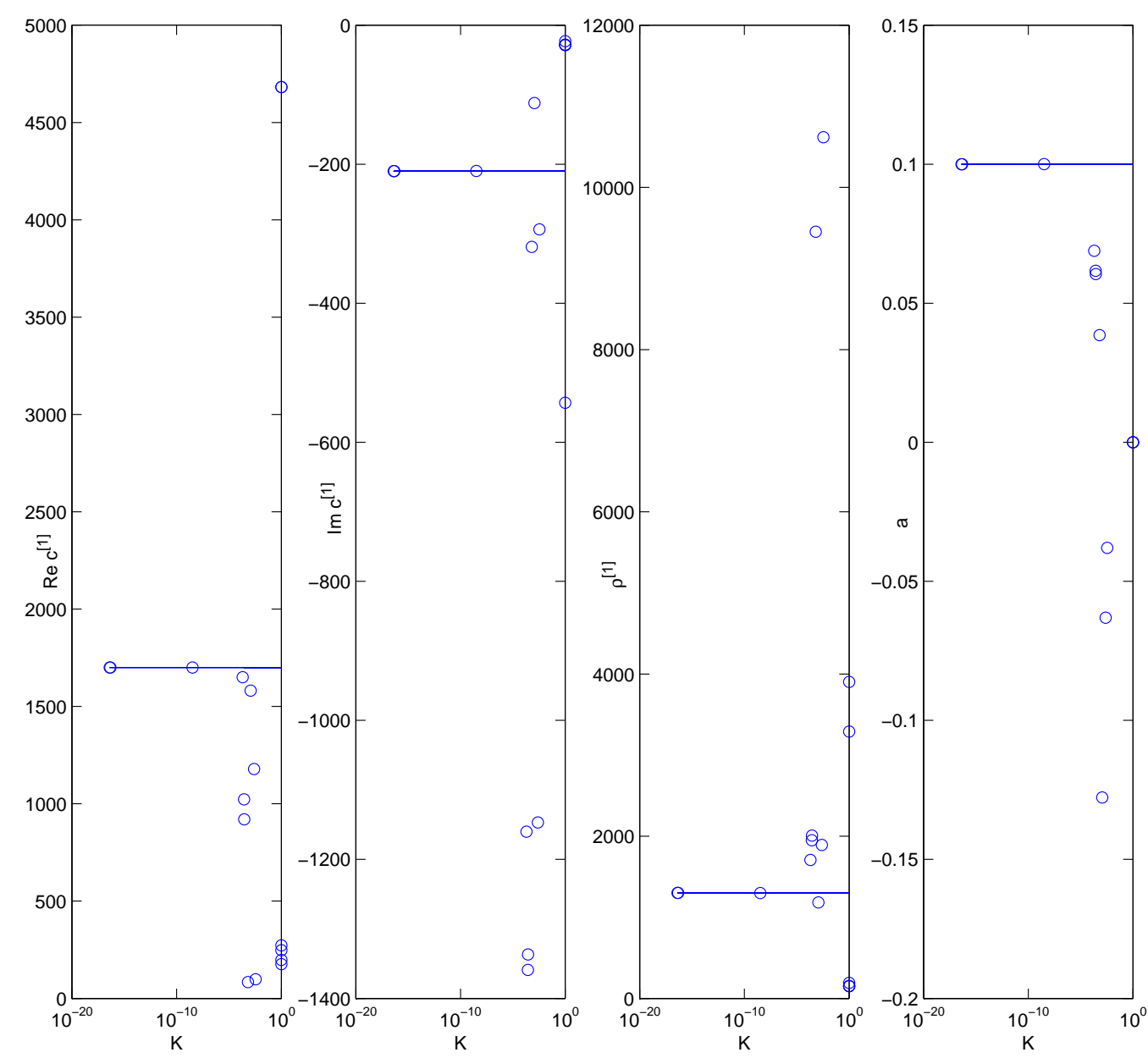

Figure 8: Case 250213-0830. $M F E=900$. 

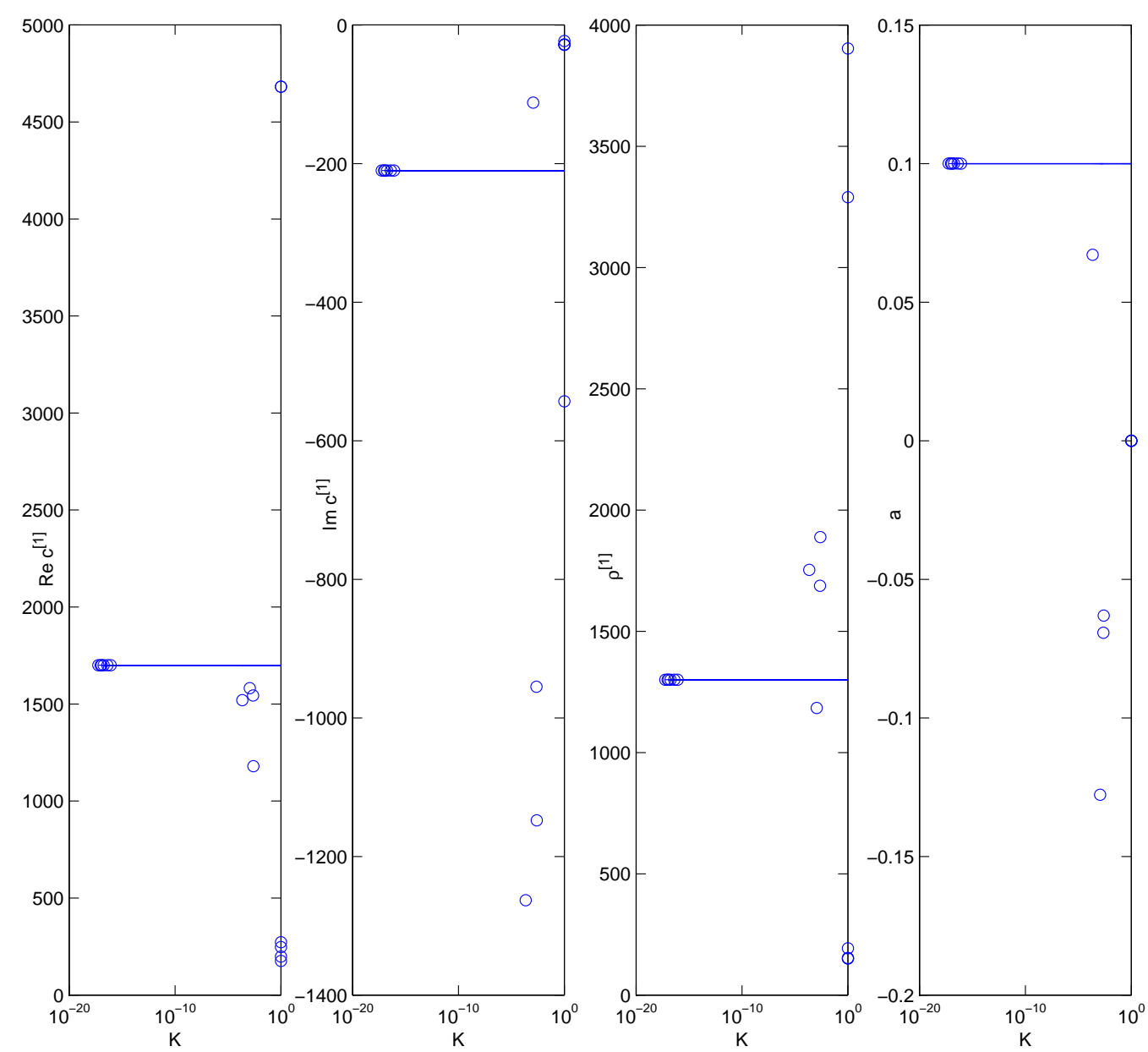

Figure 9: Case 250213-1318. $M F E=2000$. 


\subsubsection{Comments on figs. 7-9}

Figs. 7-9 show that the convergence, as a function of $M F E$, is much slower for $d=.9$ than for the small initial parameter intervals. Note that in these figures, two groups of points are visible: the first group comprises points which are on the target line, and the second group comprises the remaining points which are scattered at other locations corresponding to cost function minima that are orders of magnitude larger than those corresponding to the first group. Thus, it seems reasonable to say that the first group reflects the admissible solution(s) of the inverse problem, and using this definition, we see that even for very wide initial parameter intervals, the simplex scheme hones in on the target solution provided MFE is chosen to be sufficiently large.

These results show that it is reasonable, at least in an inverse crime situation, to apply the min min cost rule for deciding which solution to retain as the final retrieved solution.

\subsection{Indications of the manner in which the retrievals depend on the choice of excitation and sensor parameters}

Each of the following figures contains five panels within which appear as many points as the number of starting value intervals in the multistage inversion procedure. The abscissas of the graphs in each panel represent the value of an excitation or sensor parameter. The ordinates of the first four panels are as previously, whereas those of the fifth panel represent the value of the minimum of the minima of the cost functions $\mathcal{K}_{k} ; k=1,2, \ldots, N=16$ found by the multistage Simplex scheme to obtain each point on the graph. The horizontal (blue) segments designate, as before, the true values of the four to-be-retrieved parameters. All the fixed parameters are as previously.

\subsubsection{Effect of the variation of the excitation parameter $\alpha=\mathcal{A}$}



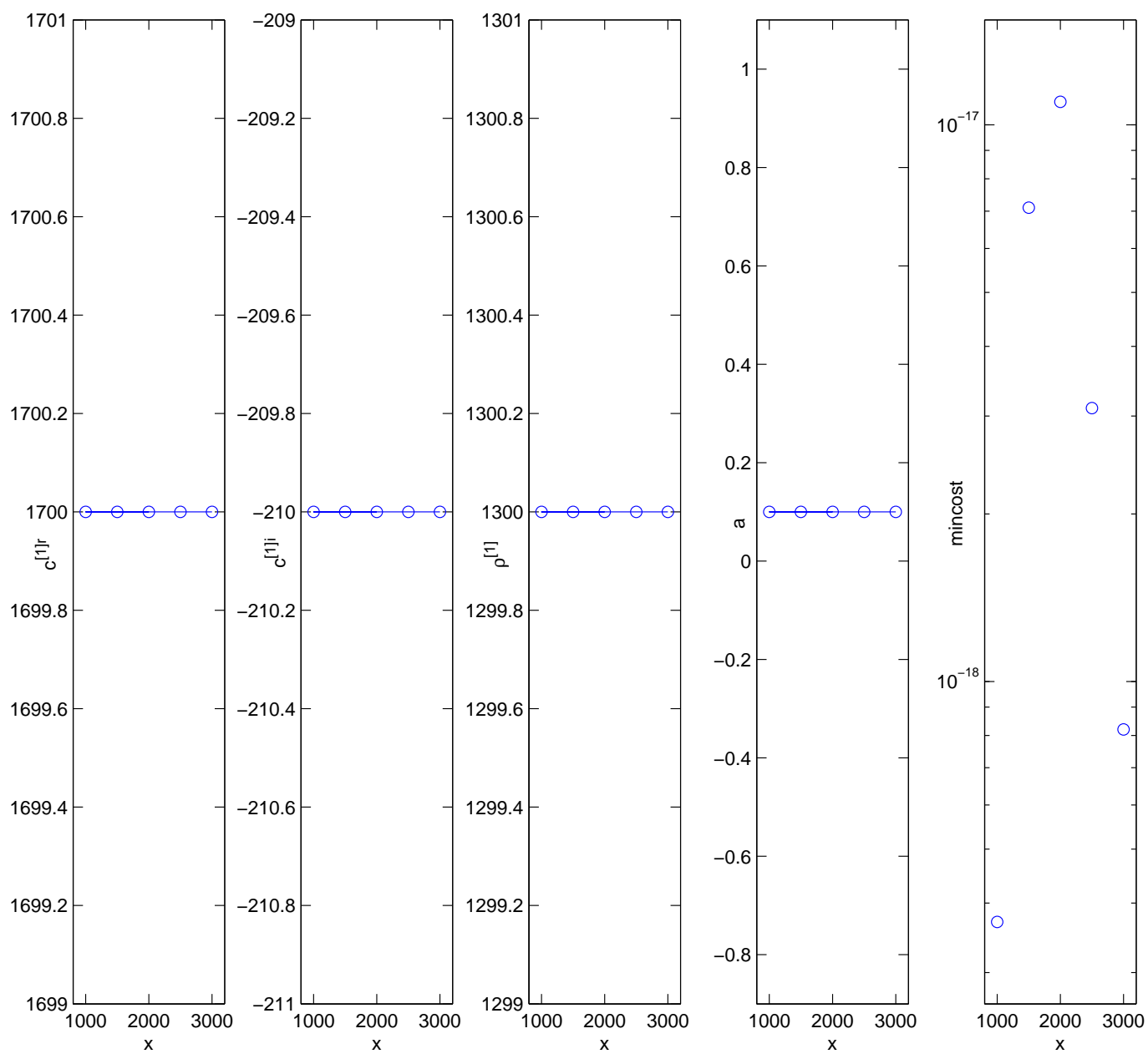

Figure 10: Case 180213-1618. The abscissas $x$ represent $\alpha=\mathcal{A} . \quad M F E=2000, \mathbf{C}^{[1] r}=$ $(170,3230,2), \mathbf{C}^{[1] i}=(-399,-21,2), \mathbf{R}^{[1]}=(130,2470,2), \mathbf{A}=(.01, .19,2), \beta=\mathcal{B}=.1$, $\boldsymbol{\theta}=(0,180,5), b=B=1 . x=1000$ is computed with $\mathbf{f}=(.003,1000,101), x=1500$ is computed with $\mathbf{f}=(.003,1500,151), \ldots, x=3000$ is computed with $\mathbf{f}=(.003,3000,301)$. 
We see that the precision of the retrievals of all five parameters is high, and insensitive to $\alpha=\mathcal{A}$.

\subsubsection{Effect of the variation of sensor parameter $b=B$}
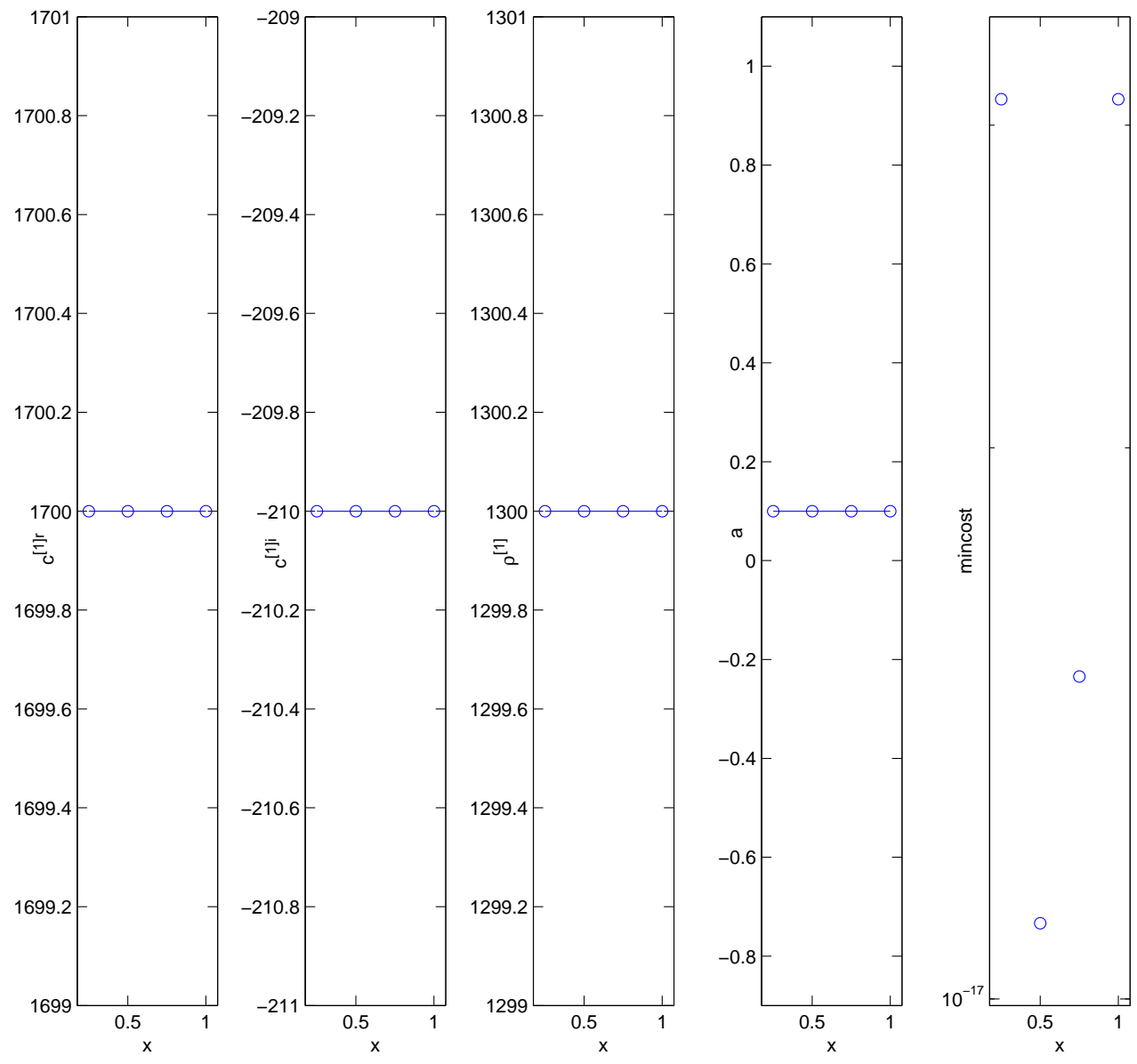

Figure 11: Case 160213-1448. The abscissas $x$ represent $b=B . \quad M F E=1000, \mathbf{f}=$ $(.003,2000,201), \mathbf{C}^{[1] r}=(500,4000,2), \mathbf{C}^{[1] i}=(-1000,-100,2), \mathbf{R}^{[1]}=(500,3000,2), \mathbf{A}=$ $(.01, .2,2), \theta^{i}=\Theta^{i}=0, \alpha=\mathcal{A}=2000, \beta=\mathcal{B}=.1, \boldsymbol{\theta}=(0,180,5)$. 
We see that the precision of the retrievals of all five parameters is high, and insensitive to $b=B$.

\subsubsection{Effect of the variation of sensor parameter $N_{\theta}$}
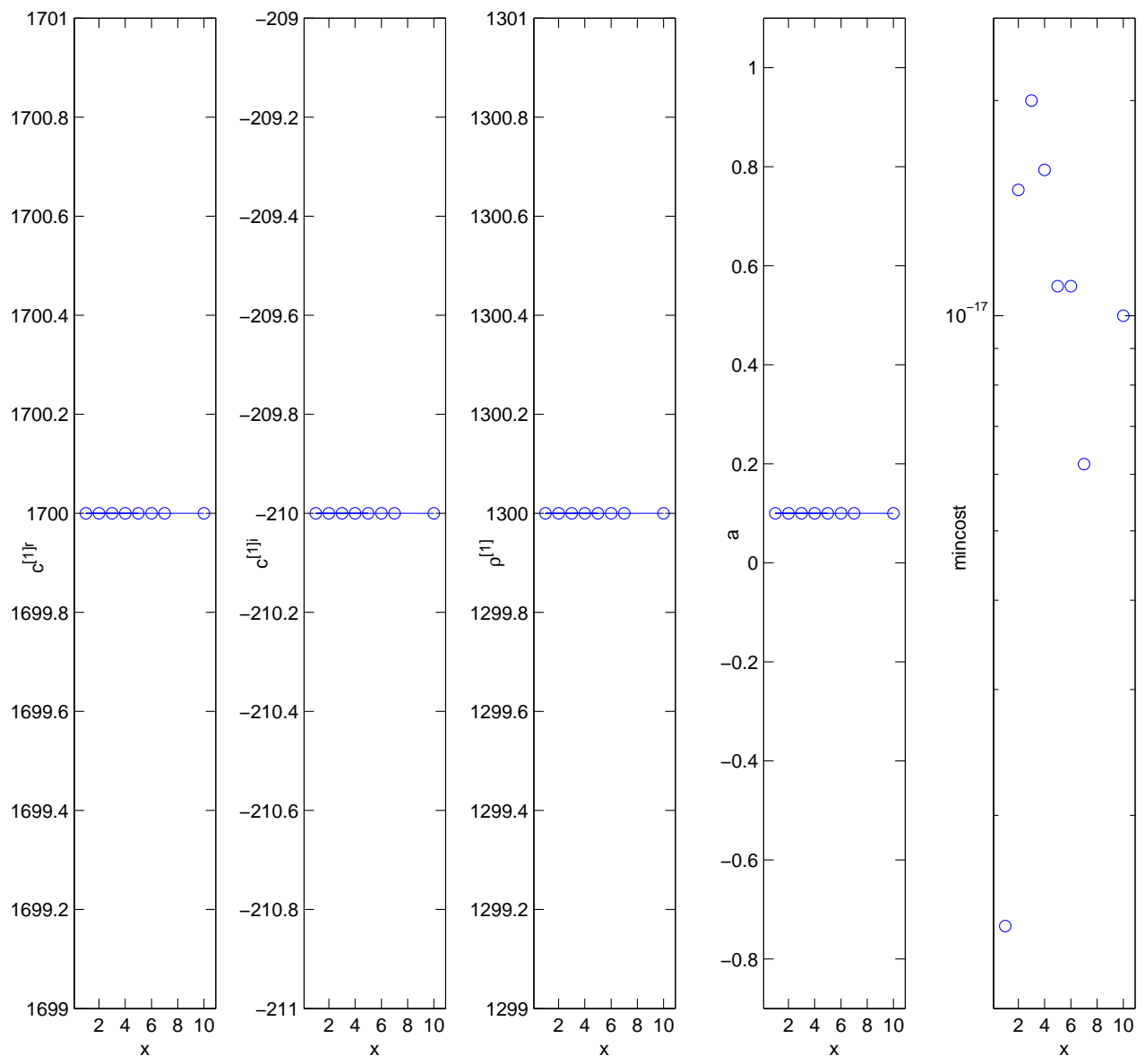

Figure 12: Case 190213-1520. The abscissas $x$ represent $N_{\theta} . \quad M F E=2000 . \quad \mathbf{C}^{[1] r}=$ $(170,3230,2), \quad \mathbf{C}^{[1] i}=(-399,-21,2), \quad \mathbf{R}^{[1]}=(130,2470,2), \quad \mathbf{A}=(.01, .19,2) . \quad \boldsymbol{\theta}=$ $\left(0,180, N_{\theta}\right)$. 
This figure indicates that the precision of the retrievals of all five parameters is high and insensitive to $N_{\theta}$.

\subsection{Final comments on the numerical results for the inverse crime situation}

We have shown that, in the inverse crime situation, it is possible, by applying the zerothorder (min min cost) regularization, to generate a unique solution to the parameter retrieval problem, provided the cost functional minimization scheme (here, the Nelder-Mead algorithm) is allowed to do its work to completion (i.e., by taking $M F E$ (in the Matlab version) to be sufficiently-large).

The retrieved parameters comprising this solution turn out to be, for all practical purposes, the true parameters, i.e., the inverse crime enables an error-free retrieval (i.e., the value of the corresponding cost functional can be of the order of $10^{-18}$, and the cumulative retrieval error of the order of $10^{-9}$ ) of the sought-for parameters.

Naturally, the amount of computations necessary to attain this result is all the larger, the less knowledge one has at the outset of the true values of the parameters, but however small be this knowledge, one succeeds in finding the true solution after a sufficient amount of computations. This is true, whatever: the characteristics of the excitation pulse, and the number of sensors as well as their distance from the scattering body.

It is important to realize that these conclusions hold only in the very specific, unrealistic, situation in which the assumed values of the priors $(\mathbf{Q})$ are the same as their true values (q). Therefore, in what follows, we address the question of how the the precision, of the retrievals is affected by discordances between $\mathbf{Q}$ and $\mathbf{q}$. We shall encounter cost functions with authentic local minima which constitute sources of confusion in the selection process of the 'best' solution, and obtain the latter in a natural, systematic manner via first- and second-order regularization schemes.

\section{$7 \quad$ Numerical results for the non-inverse crime situation in which there is an uncertainty on the prior relative to the wavespeed in the host medium}

All the parameters (except those mentioned specifically) are the same as in sect. 6. Here we examine the situation in which $C^{[0]} \neq c^{[0]}$.

\subsection{Effect of the variation of $M F E$}

We consider, in figs. 13-16, a case of mild discordance of the wavespeed in the host medium, $c^{[0]}=1500, C^{[0]}=1515$, corresponding to a relative prior uncertainty of $1 \%$. The variation of the retrievals with respect to the Simplex parameter $M F E$ is of primary concern here, but 
the successive figures also account for increasing initial to-be-retrieved parameter interval widths. The ordinates are relative to the four to-be-retrieved parameters. The abscissas are the values of the minimum of the cost functional $\mathcal{K}$ (designated by $\mathrm{K}$ ).

7.1.1 Initial parameter intervals corresponding to $d_{R_{j}}=d=.5 ; j=1,2,3,4$.
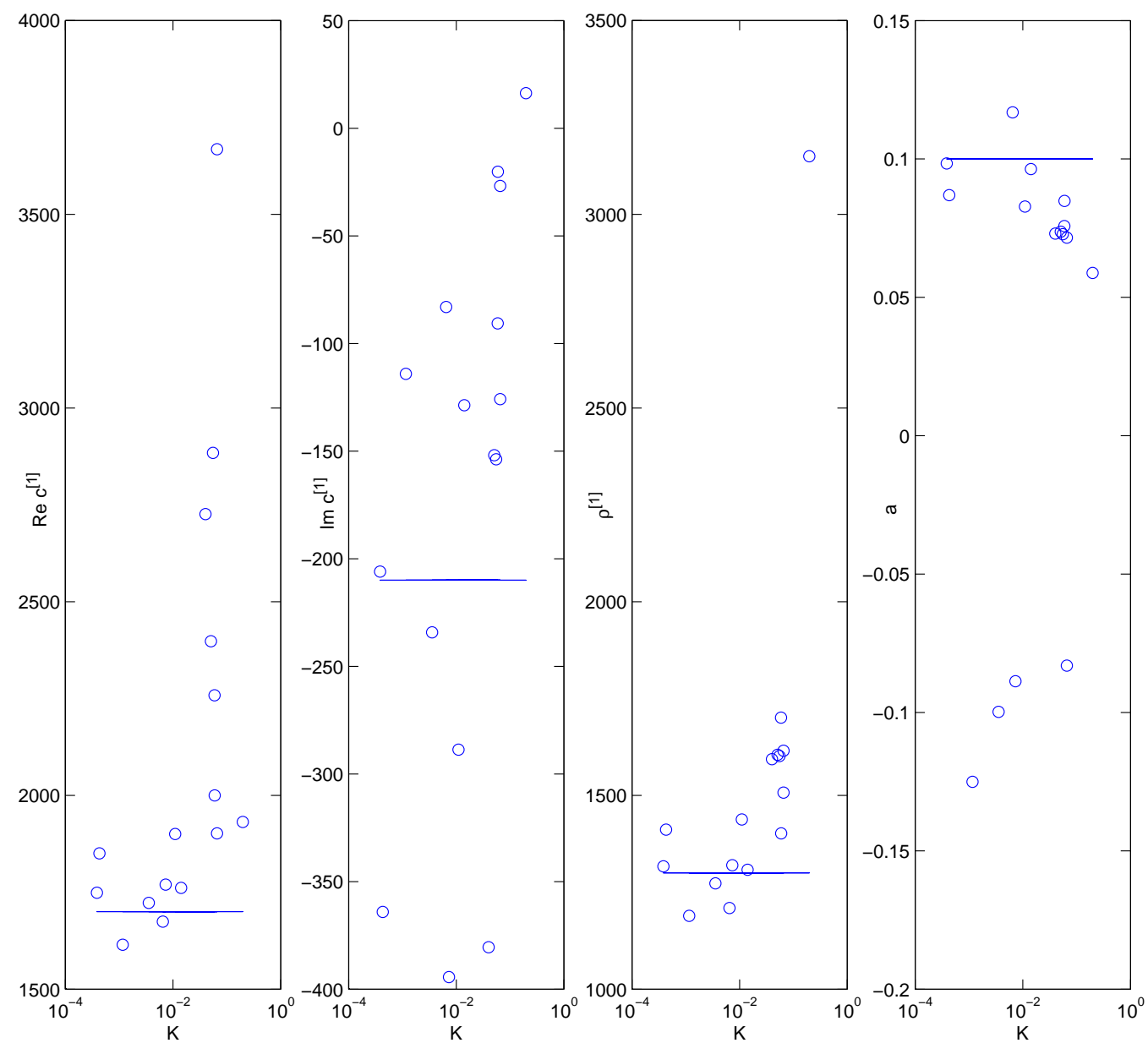

Figure 13: Case 250213-1503. $M F E=100$. 

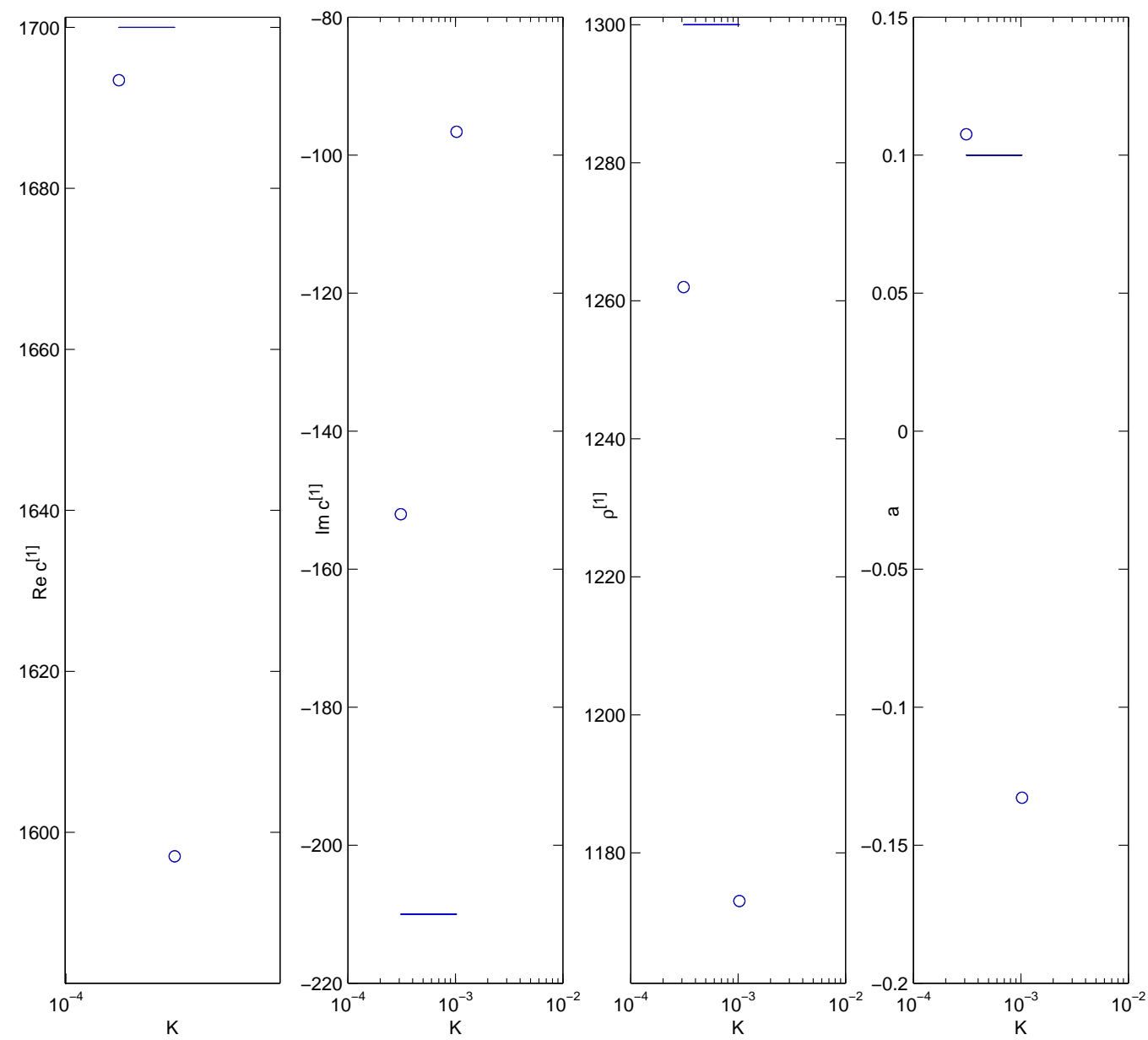

Figure 14: Case 250213-1619. $M F E=1000$. 
7.1.2 Initial parameter intervals corresponding to $d_{R_{j}}=d=.9 ; j=1,2,3,4$.

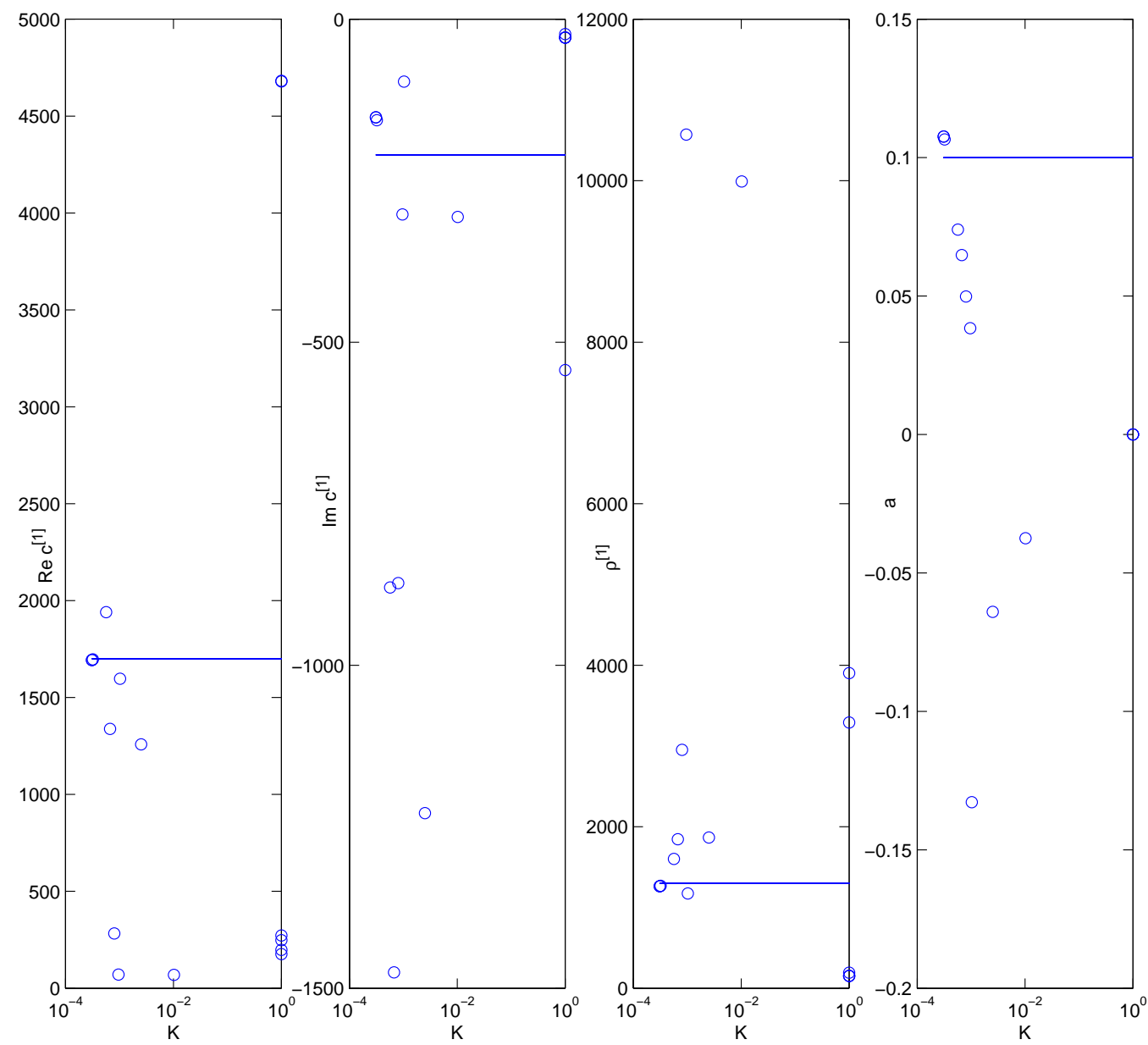

Figure 15: Case 250213-1953. $M F E=900$. 

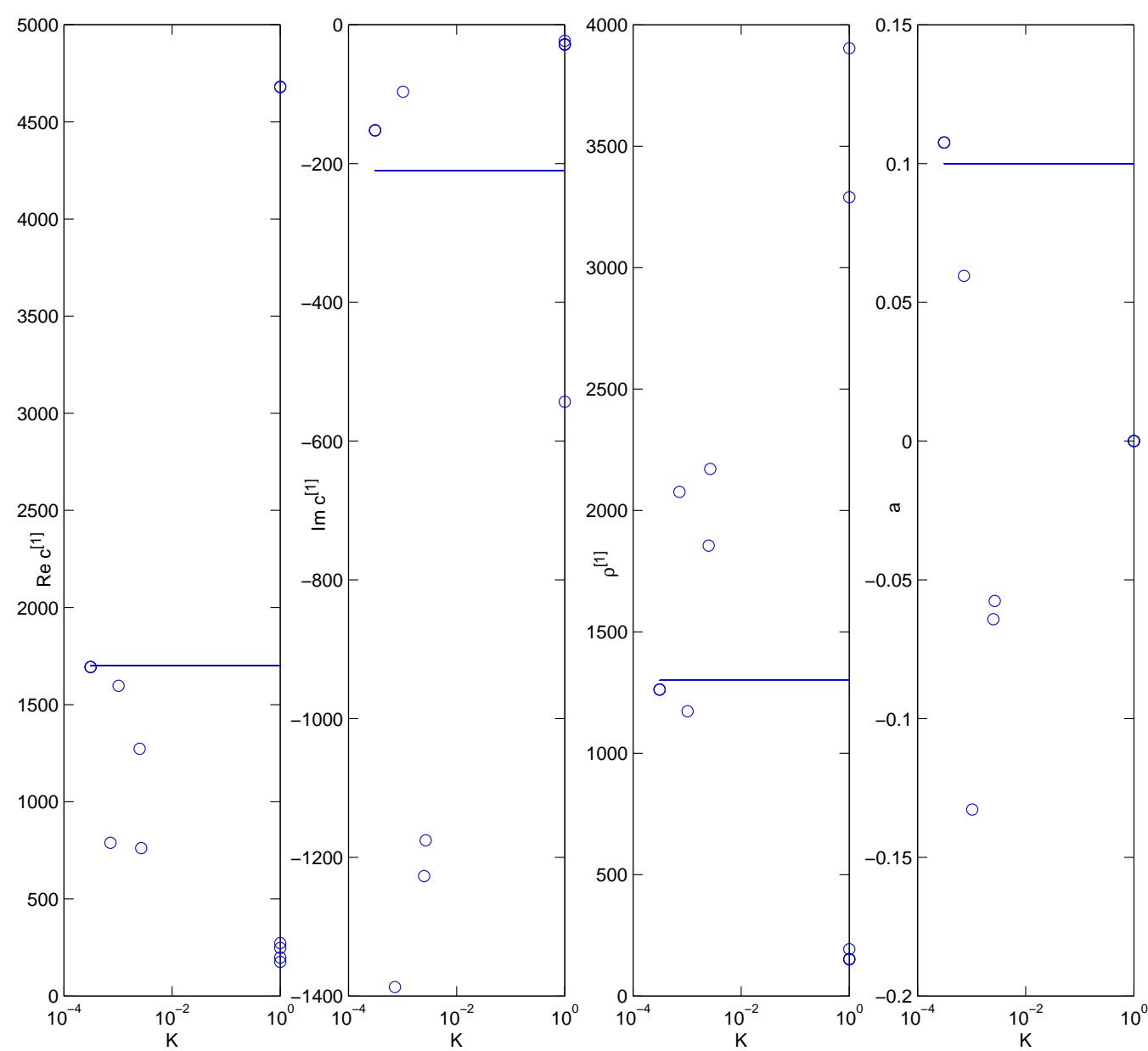

Figure 16: Case 250213-2317. $M F E=2000$. 


\subsubsection{Comments on figs. 13-16}

Figs. $13-16$ show, in the case in which the prior $C^{[0]}$ is different by $1 \%$ from the true value $c^{[0]}$, that provided $M F E$ is taken large enough, the simplex algorithm gives rise to convergence towards a small number of apparently-admissible solutions, all the faster, the narrower is the initial parameter interval. However, some of these solutions are of suspicious validity since they correspond to negative radii $a$, probably caused by the Simplex algorithm getting stuck in local minima.

In practice, one doesn't know where the targets are in the search space, but he does know that the radius $a$ of the cylinder should be a positive number. This suggests that the zerothorder regularization rule should be replaced by the first order regularization rule: to select the 'best' solution, reject clearly-unrealistic solutions (such as those for which $a$ is negative) if they exist, and finally select, among the remaining solutions, the one corresponding to $\min \min \mathcal{K}$.

We find, by applying this first-order regularization rule, that in both cases $d=.5, .9$, the relative retrieval errors are: $\varepsilon_{c^{[1] r}}=-.0039, \varepsilon_{c^{[1] i}}=-.276, \varepsilon_{\rho^{[1]}}=-.029, \varepsilon_{a}=.076$ and $\kappa=.3849$. Thus, a relative prior error of only $1 \%$ gives rise to a per cent cumulative retrieval error of $38 \%$ which is quite large. Similar effects of prior uncertainty were reported in [22], albeit for single-parameter retrieval and a different canonical scattering body.

\subsection{Variation of $d$ for larger discordances between $C^{[0]}$ and $c^{[0]}$}

Fig. 17, which was obtained by invoking zeroth-order regularization, shows, in the case in which the prior $C^{[0]}$ is different by $3 \%$ from the true value $c^{[0]}$, that the Simplex algorithm gives rise to what appears to be a form of bistability of the retrievals as a function of initial parameter uncertainty $d$.

The application of first-order regularization (i.e., elimination of the points for which $a$ is negative) enables the elimination of this apparent bistability. 


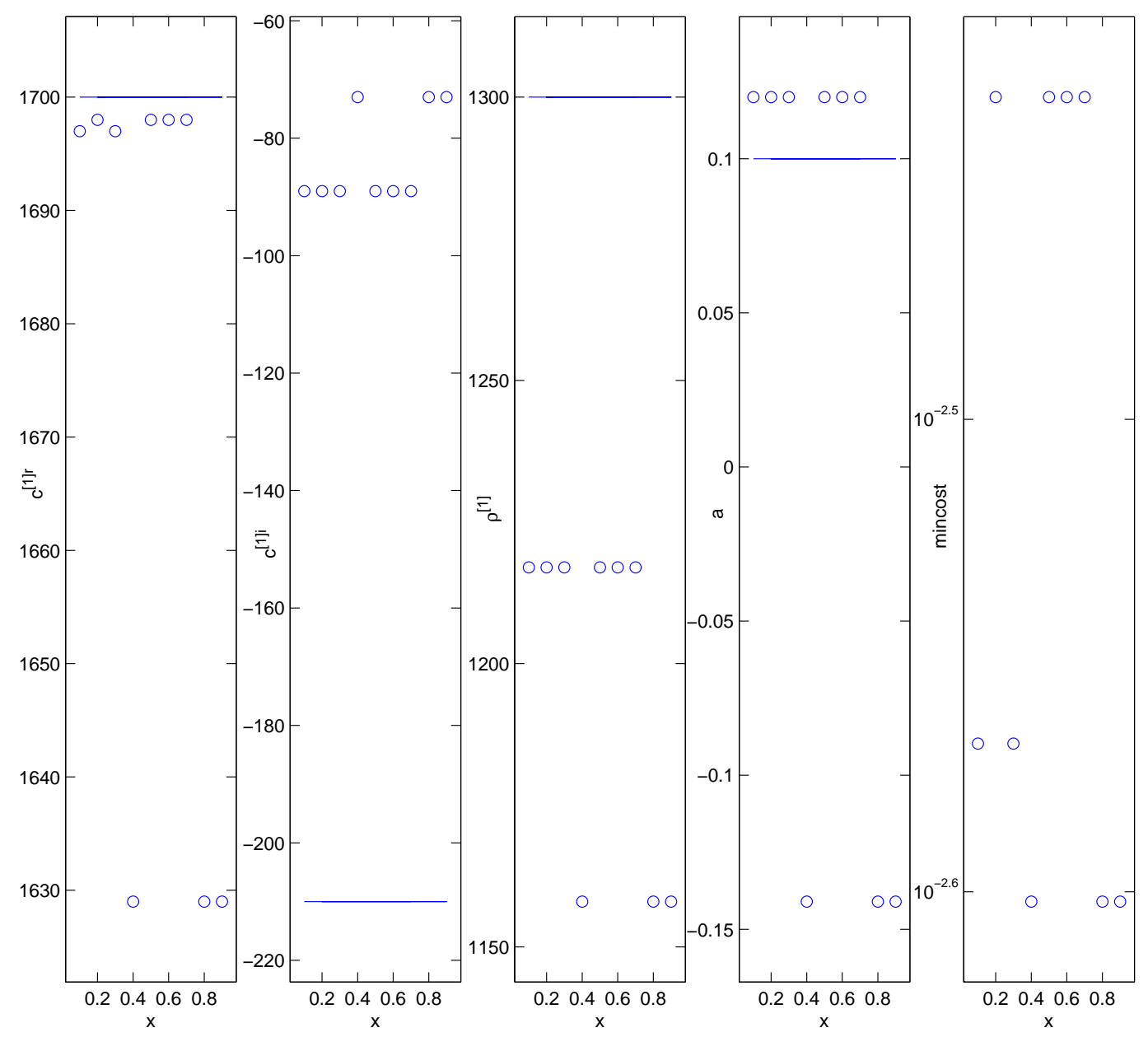

Figure 17: Case 140213-1115. The abscissas $x$ represent $d_{R_{j}}=d ; j=1,2,3,4$. $M F E=$ 2000, $c^{[0]}=1500, C^{[0]}=1545, \rho^{[0]}=R^{[0]}=1000, \theta^{i}=\Theta^{i}=0, \alpha=\mathcal{A}=2000, \beta=\mathcal{B}=.1$, $b=B=1, \boldsymbol{\theta}=(0,180,2)$. 


\subsection{Effect of $C^{[0]}$ prior uncertainty on retrieval error}

We now vary the discordance between $c^{[0]}=1500$ and $C^{[0]}$ in order to observe the effect on the precision of the retrievals.

Fig. 18, which was obtained by the min min cost (zeroth-order) rule, tells us that the increasing $C^{[0]}$ prior discordance has a nonlinear effect on the outcome of the various parameter retrievals, accompanied by the appearance of what seems to be a bifurcation of solutions between $C^{[0]}=1530$ and $C^{[0]}=1545$.

The appearance of the second branch in this figure once again raises the question of whether or not we are in the presence of an authentic instability.

It is generally admitted that nonlinear inverse problems are ill posed (Hadamard, 1923; Banks \& Kunisch, 1989; Bui, 1993; Isakov, 2009) which means that the solution either does not exist, or is not unique and is unstable in the case of existence. Instability is usually defined (Banks \& Bihari, 2001) by retrievals not being continuously-dependent on variations of the data. Herein (since the data is fixed), instability is characterized by the retrievals not being continuously-dependent on variations of model uncertainties (i.e., those of the priors). The question is now whether first-order regularization is adequate to deal with this instability, as was done in sect. 7.2. 

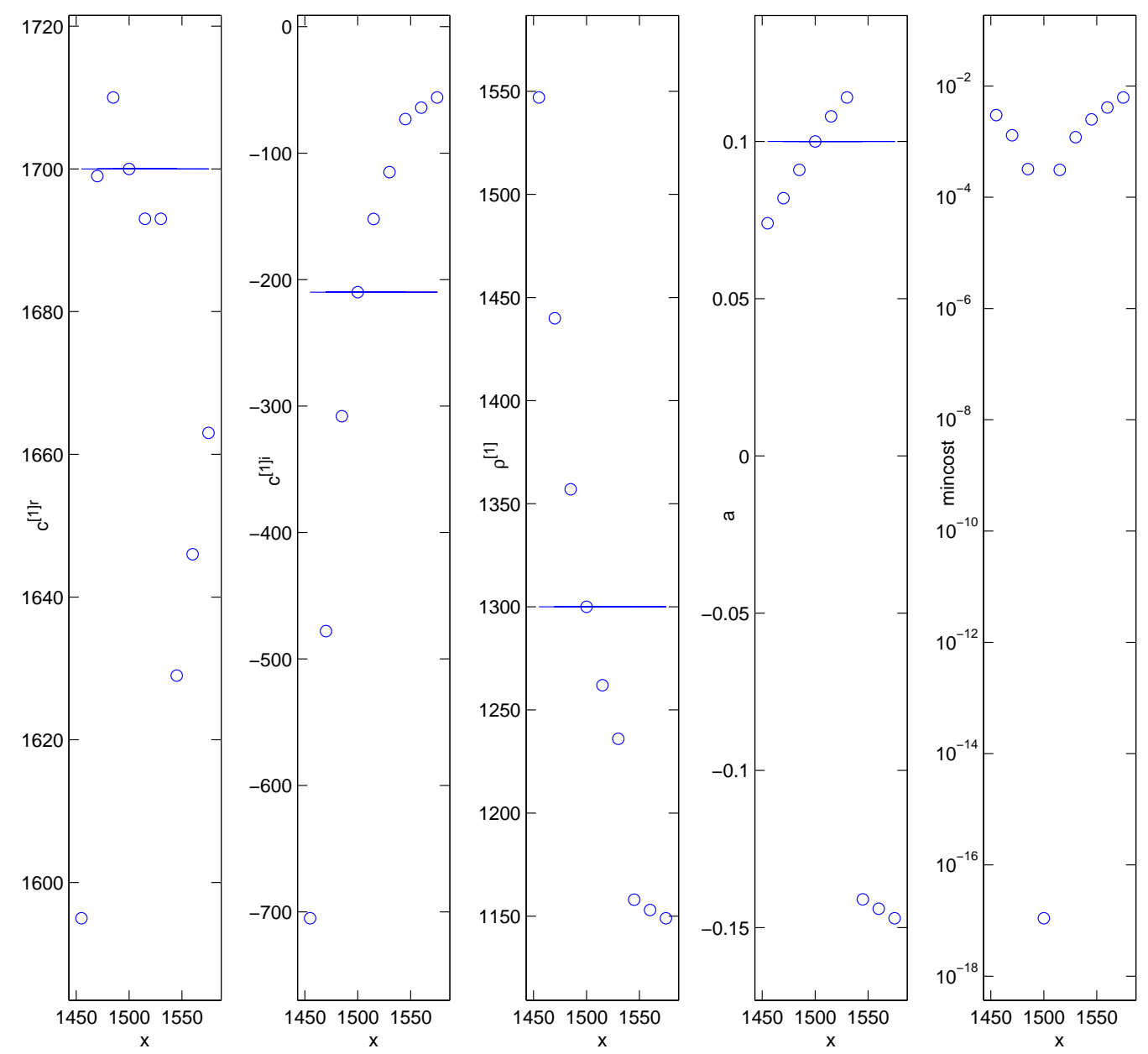

Figure 18: Case 170213-1153. The abscissas $x$ represent $C^{[0]} . d_{R_{j}}=d=.9 ; j=1,2,3,4$. $M F E=2000, c^{[0]}=1500, \rho^{[0]}=R^{[0]}=1000, \theta^{i}=\Theta^{i}=0, \alpha=\mathcal{A}=2000, \beta=\mathcal{B}=.1$, $b=B=1, \boldsymbol{\theta}=(0,180,5)$. 
To answer this question requires a microscopic analysis of the solutions generated by the Simplex algorithm, for the values $C^{[0]}=1530$ on the first branch, and $C^{[0]}=1545$ on the second branch in fig. 18. Figs. 19-20 enable this analysis.
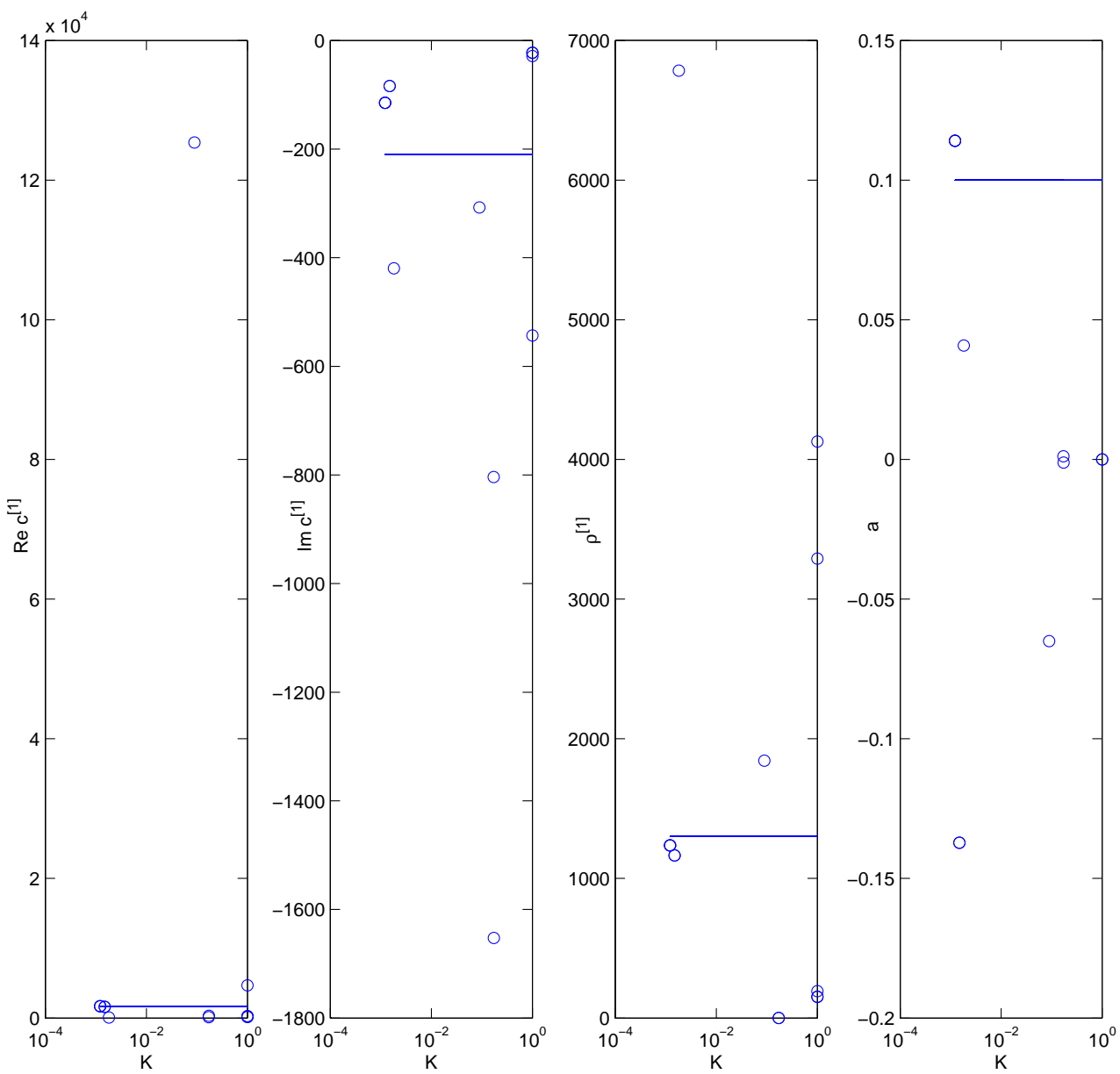

Figure 19: Case 050313-1007. The abscissas $x$ represent $\min \mathcal{K}_{k} . d_{R_{j}}=d=.9 ; j=1,2,3,4$. $M F E=2000 . c^{[0]}=1500 . C^{[0]}=1530 . \rho^{[0]}=R^{[0]}=1000 . \theta^{i}=\Theta^{i}=0 . \alpha=\mathcal{A}=2000$. $\beta=\mathcal{B}=.1 . b=B=1 . \boldsymbol{\theta}=(0,180,5)$. 

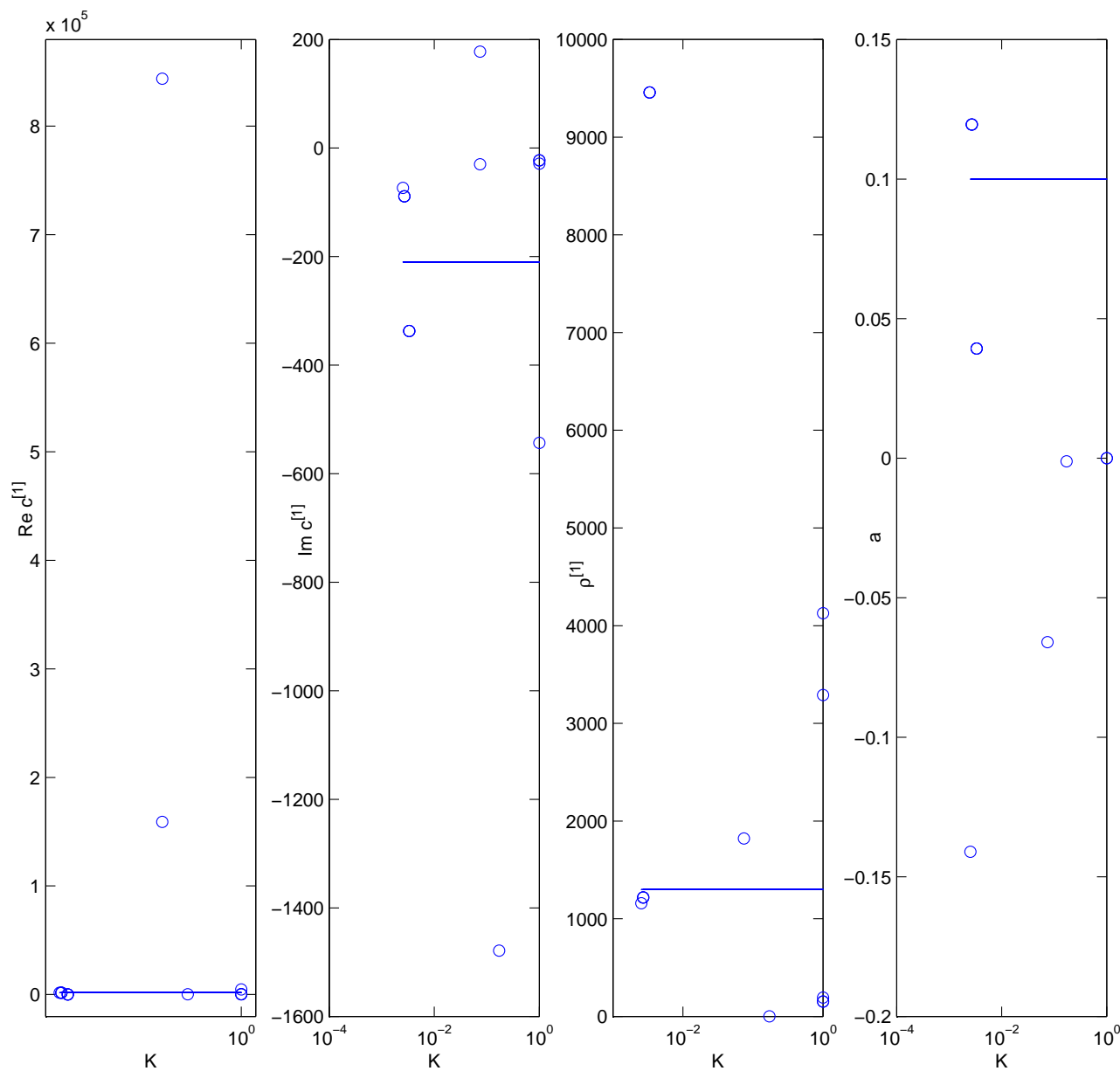

Figure 20: Case 040313-1001. The abscissas $x$ represent $\min \mathcal{K}_{k} . d_{R_{j}}=d=.9 ; j=1,2,3,4$. $M F E=2000 . c^{[0]}=1500 . C^{[0]}=1545 . \rho^{[0]}=R^{[0]}=1000 . \theta^{i}=\Theta^{i}=0 . \alpha=\mathcal{A}=2000$. $\beta=\mathcal{B}=.1 . b=B=1 . \boldsymbol{\theta}=(0,180,5)$. 
In each of these figures, we observe that the Simplex algorithm generates two sets of solutions corresponding to two sets of $\min \mathcal{K}$ that are separated from one another by at least one order of magnitude. After eliminating the solutions associated with the larger min $K$ we have to contend with three points in figs. 19-20.

\begin{tabular}{|c|c|c|c|c|}
\hline$\left[\mathbf{R}_{b}, \mathbf{R}_{e}\right]$ & $\mathbf{r}$ & $\widetilde{\mathbf{r}}$ at point 1 & $\widetilde{\mathbf{r}}$ at point 2 & $\widetilde{\mathbf{r}}$ at point 3 \\
\hline$[170,3230]$ & 1700 & 1693 & 1613 & $\mathbf{8 8 . 4 5}$ \\
{$[-399,-21]$} & -210 & -114.9 & -83.97 & $\mathbf{- 4 1 9 . 6}$ \\
{$[130,2470]$} & 1300 & 1236 & 1165 & $\mathbf{6 7 8 4}$ \\
{$[.01, .19]$} & .1 & .1141 & -.1372 & .04075 \\
\hline $\min \mathcal{K}$ & & .002542 & .002701 & .003337 \\
& & & & \\
\hline
\end{tabular}

Table 4: Three admissible retrievals of $c^{[1]}$ for $C^{[0]}=1530$. The boldfaced numbers correspond to a retrieved parameter that is not within the corresponding initial parameter interval (first column of the table).

In table 4 we now adopt the second-order regularization rule briefly alluded-to in sect. 2.6: any solution $\widetilde{\mathbf{r}}$, a member of which is outside the corresponding member of $\left[\mathbf{R}_{b}, \mathbf{R}_{e}\right]$, must be rejected, and if more than one solution persists after this rejection procedure, then the one corresponding to $\min \min \mathcal{K}$ is chosen to be the final (supposedly-correct) solution.

The application of this rule enables us to eliminate the solutions associated with points 2 and 3 . Thus, the retained solution is the one at point 1, which happens to correspond to the smallest value of $\min \mathcal{K}$.

Note that the manner of selecting the correct solution by the first-order regularization rule (i.e., elimination of the retrievals for which $a$ is negative) is not sufficiently discriminative since it does not enable to exclude the solution in the last column of table 4. 


\begin{tabular}{|c|c|c|c|c|}
\hline$\left[\mathbf{R}_{b}, \mathbf{R}_{e}\right]$ & $\mathbf{r}$ & $\widetilde{\mathbf{r}}$ at point 1 & $\widetilde{\mathbf{r}}$ at point 2 & $\widetilde{\mathbf{r}}$ at point 3 \\
\hline$[170,3230]$ & 1700 & 1629 & 1697 & $\mathbf{5 9 . 4 5}$ \\
{$[-399,-21]$} & -210 & -73.28 & -88.89 & -337 \\
{$[130,2470]$} & 1300 & 1158 & 1217 & $\mathbf{9 4 5 9}$ \\
{$[.01, .19]$} & .1 & -.141 & .1196 & .03926 \\
\hline $\min \mathcal{K}$ & & .002542 & .002701 & .003337 \\
& & & & \\
\hline
\end{tabular}

Table 5: Three admissible retrievals of $c^{[1]}$ for $C^{[0]}=1545$. The boldfaced numbers correspond to a retrieved parameter that is not within the corresponding initial parameter interval (first column of the table).

In table 5 we once again adopt the second-order regularization strategy. This enables us to eliminate the solutions at points 1 and 3. Thus, the retained solution is the one at point 2, even though (contrary to the case in table 4) it does not correspond to the smallest value of $\min \mathcal{K}$.

Note that, once again, the manner (first-order regularization) of selecting the correct solution solely by elimination of the retrievals for which $a$ is negative is not sufficiently discriminative since it does not enable to exclude the solution in the last column of table 5 .

Thus, it appears that what, in fig. 18, distinguishes the 'solutions' of the two branches is that the first (left-hand) branch corresponds to solutions associated with min min $\mathcal{K}_{k}$, whereas the second (right-hand) branch corresponds to solutions for which $\min \mathcal{K}_{k}$ is not minimal.

The selection of the correct solution solely on the basis of $\min \min \mathcal{K}_{k}$ is not in contradiction with the present manner since, in applications of the $\min \min \mathcal{K}_{k}$ rule, no 'solution' corresponded to one or several retrieved parameters being outside of the corresponding initial parameter search intervals, which is the situation that prevails either when one commits the inverse crime or when the discordance between assumed prior(s) $\mathbf{Q}$ and the corresponding true prior(s) $\mathbf{q}$ is (are) small.

Otherwise (case of relatively-large discordance between $\mathbf{Q}$ and $\mathbf{q}$ ), we are no longer in the (inverse crime) situation characterized by a cost functional with a single (global) minimum, but rather in one marked by the appearance of more-than-one (local) minima which are a source of non-uniqueness and instability in the inversion procedure. We have shown, that even in such cases, it is possible, by adopting the second-order regularization procedure to eliminate, in algorithmic manner, all but one solution.

\subsubsection{The revised graph of retrieved parameters versus $\mathcal{C}^{[0]}$}

From the results of tables 4-5 it is now clear that the second branch in fig. 18 is anomalous due to the fact that the retrievals to which they correspond are not correct (in the sense of 
the second-order regularization rule). By replacing the points on this branch by the correct points (found e.g., in table 5), we obtain the new, revised, graph of fig. 21 and notice that all signs of instability have disappeared. From this graph we find that a prior uncertainty
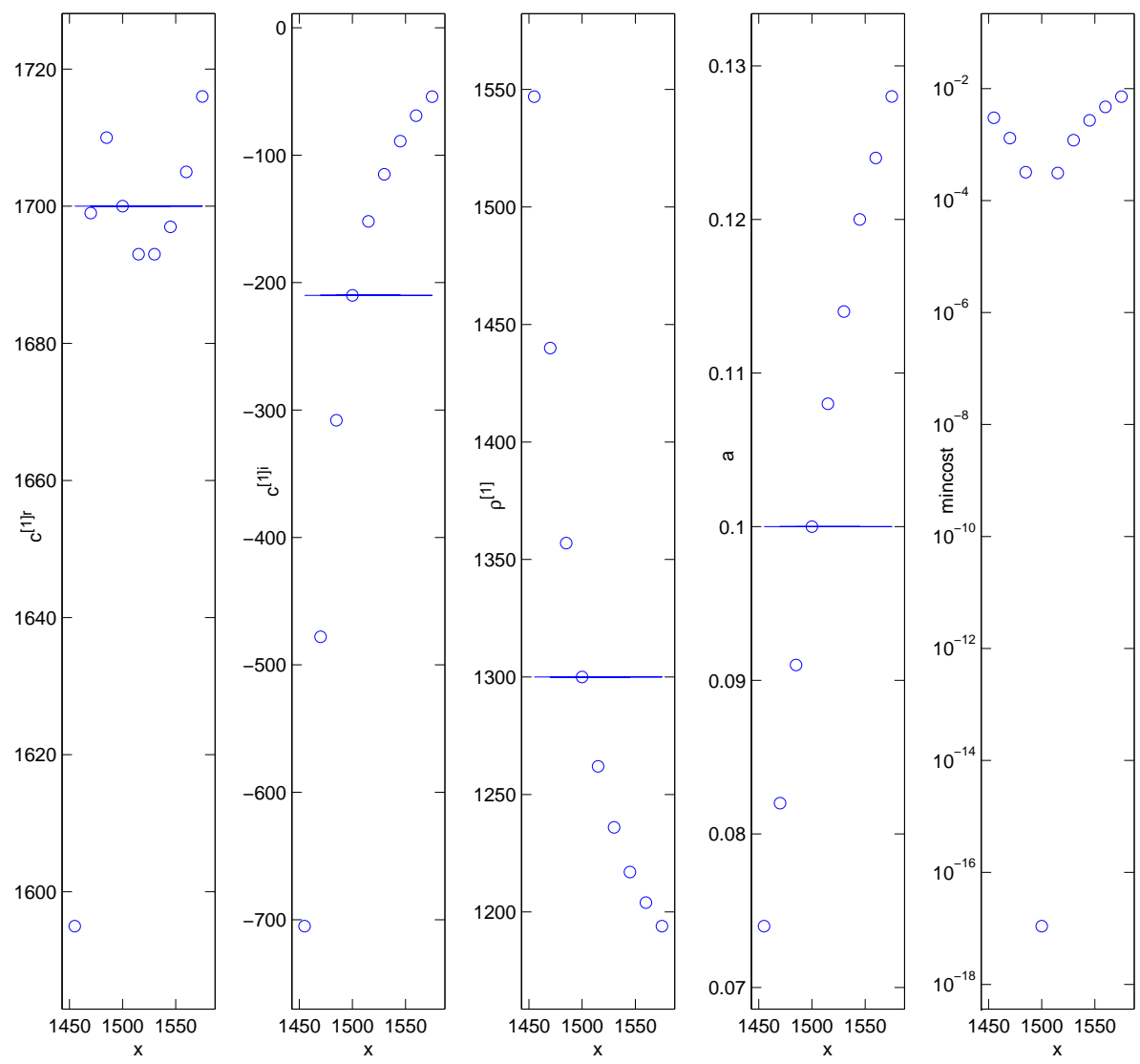

Figure 21: Case 040313-1242. Revised graph of fig. 18 after application of the second-order regularization. The abscissas $x$ represent $C^{[0]} . d_{R_{j}}=d=.9 ; j=1,2,3,4 . \quad M F E=2000$, $c^{[0]}=1500, \rho^{[0]}=R^{[0]}=1000, \theta^{i}=\Theta^{i}=0, \alpha=\mathcal{A}=2000, \beta=\mathcal{B}=.1, b=B=1$, $\boldsymbol{\theta}=(0,180,5)$.

$\delta_{C[0]}=-3 \%$ generates a cumulative retrieval error of $\kappa=28.5 \%$, whereas a prior uncertainty $\delta_{C[0]}=+3 \%$ generates a cumulative retrieval error of $\kappa=84.2 \%$. These errors, particularly the second one, are very large compared to the prior uncertainties to which they give rise. Similar effects of prior uncertainty were reported in [22], albeit for single-parameter retrieval and a different canonical body. 


\section{Numerical results concerning the effect of the other prior uncertainties on retrieval error}

\subsection{Effect of $\mathcal{R}^{[0]}$ prior uncertainty}
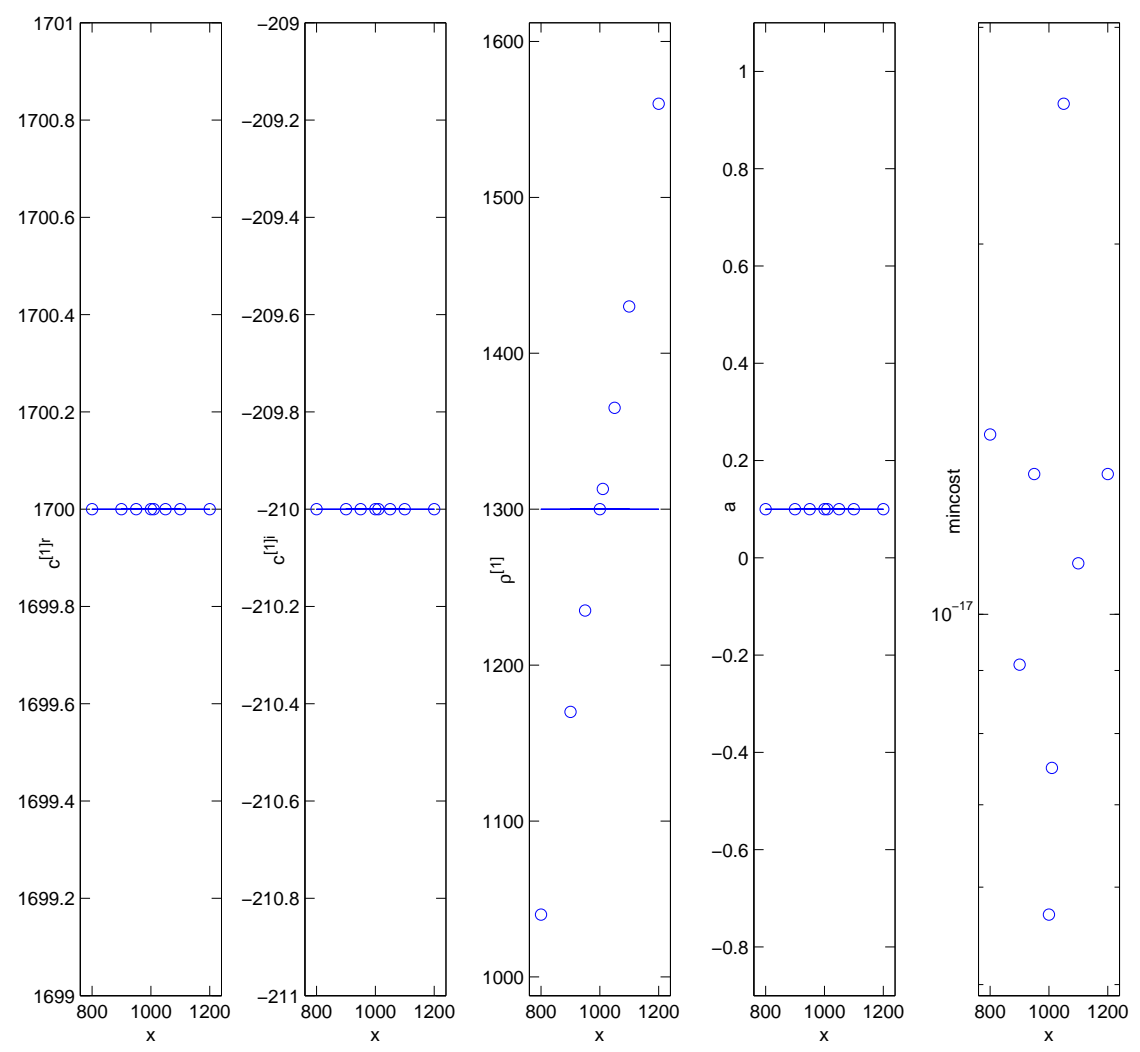

Figure 22: Case 140213-1006. The abscissas $x$ represent $\mathcal{R}^{[0]} . d_{R_{j}}=d=.9 ; j=1,2,3,4$. $M F E=2000, c^{[0]}=C^{[0]}=1500, \rho^{[0]}=1000, \theta^{i}=\Theta^{i}=0, \alpha=\mathcal{A}=2000, \beta=\mathcal{B}=.1$, $b=B=1, \boldsymbol{\theta}=(0,180,5)$.

Fig. 22, which was obtained using the second-order regularization, reveals that $\mathcal{R}^{[0]}$ prior uncertainty provokes negligible error in the retrievals of $c^{[1] r}, c^{[1] i}$, and $a$, and linearly-varying error of $\rho^{[1]}$. 


\subsection{Effect of $\mathcal{A}$ prior uncertainty on retrieval error}

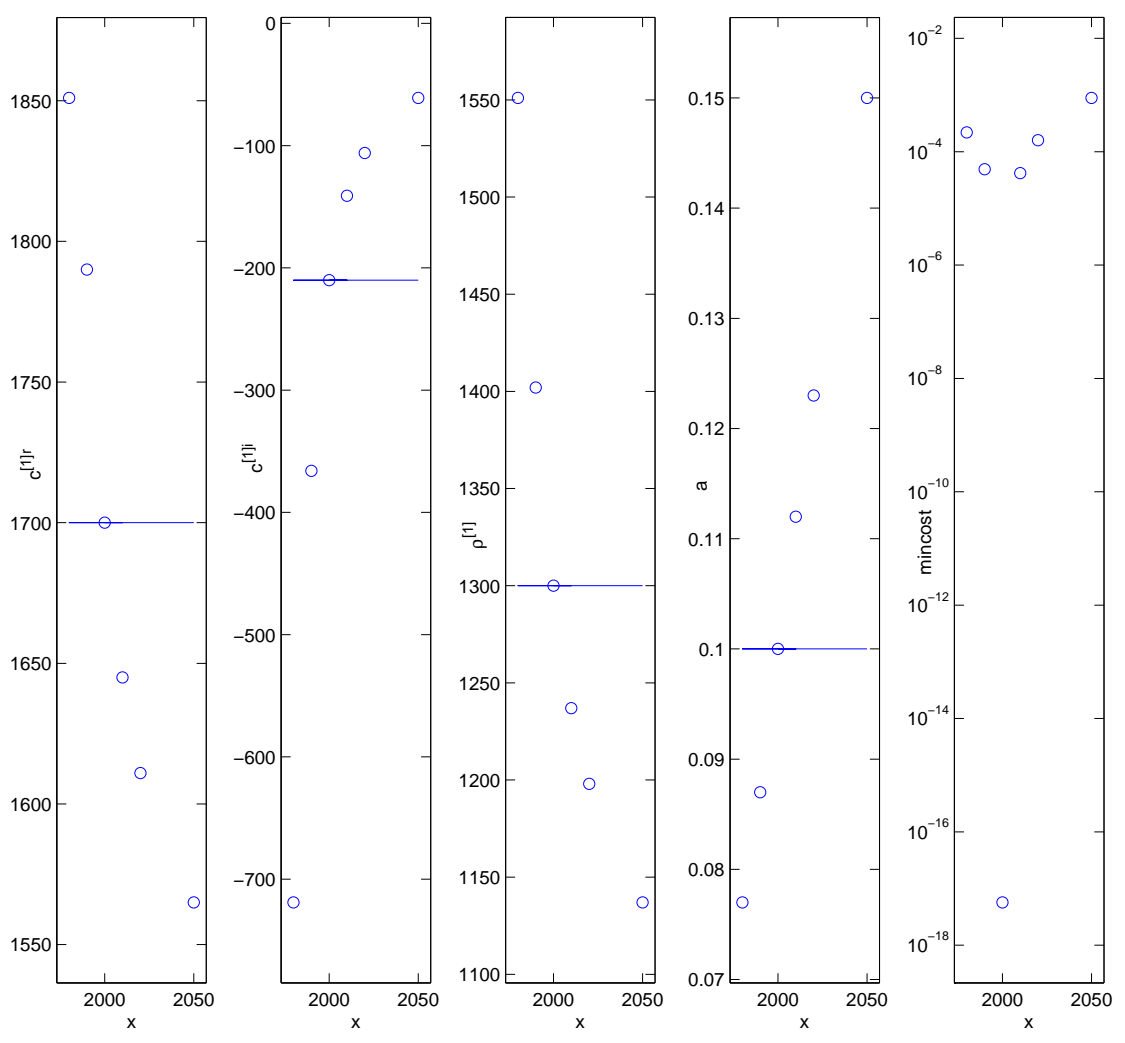

Figure 23: Case 140213-0947. The abscissas $x$ represent $\mathcal{A} . \quad d_{R_{j}}=d=.9 ; j=1,2,3,4$. $M F E=2000, c^{[0]}=C^{[0]}=1500, \rho^{[0]}=R^{[0]}=1000, \theta^{i}=\Theta^{i}=0, \alpha=2000, \beta=\mathcal{B}=.1$, $b=B=1, \boldsymbol{\theta}=(0,180,5)$.

Fig. 23, which was obtained using the second-order regularization, reveals a weaklynonlinear dependence of retrieval error on $\mathcal{A}$ prior uncertainty. For a relative prior uncertainty $\delta_{\mathcal{A}}=-.01$, the relative retrieval errors are $\varepsilon_{c^{[1] r}}=.0888, \varepsilon_{c^{[1] i}}=2.424, \varepsilon_{\rho^{[1]}}=.193$, $\varepsilon_{a}=-.230$, and for $\delta_{\mathcal{A}}=+.025$, the relative retrieval errors are $\varepsilon_{c^{[1] r}}=-.0794, \varepsilon_{c^{[1] i}}=$ $-.7095, \varepsilon_{\rho^{[1]}}=.1931, \varepsilon_{a}=0.5000$. Thus, a prior uncertainty $\delta_{\mathcal{A}}=-1 \%$ provokes a cumulative parameter retrieval error $\kappa=293.6 \%$ and $\delta_{\mathcal{A}}=2.5 \%$ gives rise to a cumulative parameter retrieval error $\kappa=148.2 \%$. Both of these errors are extremely large compared to the corresponding prior uncertainties $\delta_{\mathcal{A}}$. 


\subsection{Effect of $\mathcal{B}$ prior uncertainty}

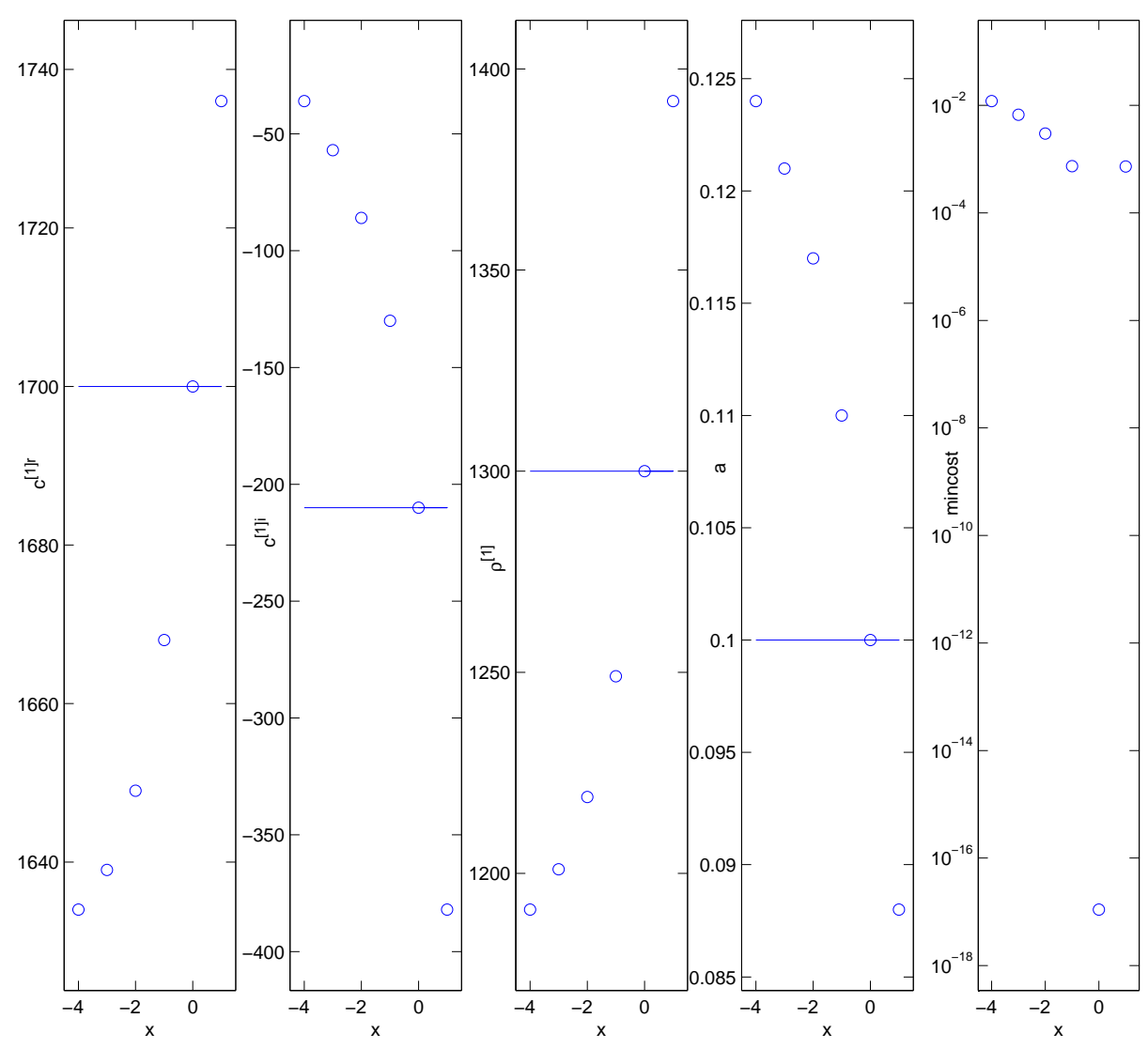

Figure 24: Case 050313-1601. The abscissas $x$ represent $\mathcal{B} . d_{R_{j}}=d=.9 ; j=1,2,3,4$. $M F E=2000, \alpha=\mathcal{A}=2000, \beta=.1, b=B=1, \boldsymbol{\theta}=(0,180,5) . x=-4$ corresponds to $\mathcal{B}=.09996, x=-3$ corresponds to $\mathcal{B}=.09997, \ldots ., x=0$ corresponds to $\mathcal{B}=.1, \ldots . ., x=3$ corresponds to $\mathcal{B}=.10003, x=4$ corresponds to $\mathcal{B}=.10004$.

Fig. 24, which was obtained using the second-order regularization, reveals that for a very small negative prior uncertainty $\delta_{\mathcal{B}}=-.0004$, the retrieval errors are: $\varepsilon_{c^{[1] r}}=-.0388$, $\varepsilon_{c^{[1] i}}=.8286, \varepsilon_{\rho^{[1]}}=-.0838, \varepsilon_{a}=.24$, and for a very small positive prior uncertainty $\delta_{\mathcal{B}}=+.0001$, the retrieval errors are: $\varepsilon_{c^{[1] r}}=.0212, \varepsilon_{c^{[1] i}}=-.8190, \varepsilon_{\rho^{[1]}}=.0708, \varepsilon_{a}=-.12$. Thus, the prior uncertainty $\delta_{\mathcal{B}}=-.04 \%$ provokes a cumulative retrieval error $\kappa=119.12 \%$ and the prior uncertainty $\delta_{\mathcal{B}}=+.01 \%$ provokes a cumulative retrieval error $\kappa=103.1 \%$. These errors are enormous compared to the corresponding prior uncertainties. 


\subsection{Effect of $\Theta^{i}$ prior uncertainty}
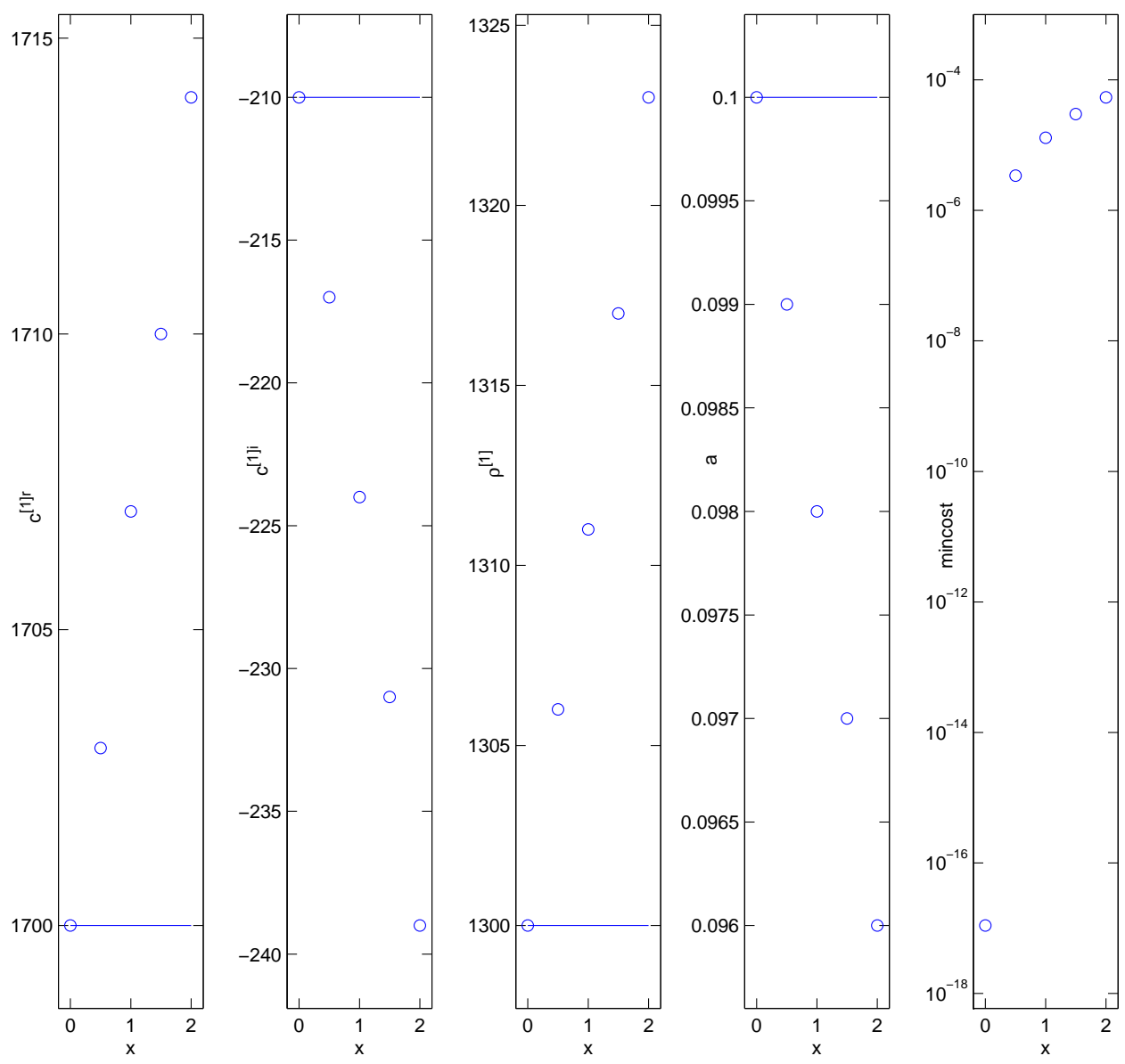

Figure 25: Case 170213-1808. The abscissa $x$ represents $\Theta^{i} . d_{R_{j}}=d=.9 ; j=1,2,3,4$. $M F E=2000, c^{[0]}=C^{[0]} 1500, \rho^{[0]}=R^{[0]}=1000, \theta^{i}=0, \alpha=\mathcal{A}=2000, \beta=\mathcal{B}=.1$, $b=B=1, \boldsymbol{\theta}=(0,180,5)$.

Fig. 25, which was obtained using the second-order regularization, manifests a quasilinear variation of error in the retrieval of the four parameters. We find for a difference of $2^{\circ}$ of the prior from its true value that the individual retrieval errors are $\varepsilon_{c^{[1] r}}=.0082, \varepsilon_{c^{[1] i}}=.1213$, $\varepsilon_{\rho^{[1}}=.0177, \varepsilon_{a}=-.04$, and the cumulative retrieval error is $\kappa=.1872$. All these errors are rather small in comparison with what was found previously for the other types of priors. 


\section{Conclusions}

The inverse problem studied herein was based on a number of choices:

$a$ - the object to be characterized was of canonical nature (homogeneous, isotropic, simple circular cylindrical form);

$b$ - the identification of this lossy-fluid object meant retrieving its one geometrical parameter (the radius) and three mechanical constitutive parameters: real and imaginary parts of the phase velocity, density;

$c$ - this was achieved by processing the scattered field time-domain data obtained by soliciting the object with a plane, transient acoustic wave;

$d$ - the processing aspect comprised two components: 1) nonlinear least squares minimization of a cost functional translating the discrepancy between synthetic signal data and trial signal data, and 2) a posteriori regularization;

$e$ - this processing was carried out in numerical manner with the help of the Nelder-Mead downhill Simplex optimization algorithm for the first component, and a simple, automatic zeroth-, first- or second-order regularization algorithm for the second component;

$f$ - the retrievals were done both in the inverse crime and non-inverse crime contexts;

$g$ - the inverse crime context was defined as the one in which the data simulation model was identical to the retrieval model and all the priors (such as phase velocity of the host fluid) incorporated in the retrieval model are identical to their counterparts in the data simulation model;

$h$ - a non-inverse crime context was defined as one in which the data simulation model was identical to the retrieval model and one or several of the priors incorporated in the retrieval model are different from their counterparts in the data simulation model.

The reasons why these choices were made were;

$A$ - choice a) enabled the direct problem of predicting the response of the object to an incident wave to be solved in quasi-analytic manner, thereby making the inversions (involving the resolution of a large number of direct problems) tractable on a laptop computer;

$B$ - choice b) derived from the requirement of multiparameter retrieval, with four to-beretrieved parameters (of heterogeneous nature) being a favorable compromise between a single to-be-retrieved parameter [22] and many to-be-retrieved parameters as for a multilayer elastic half space [41]. However, the methods and conclusions of our study should also apply to more complex, more realistic physical configurations;

$C$ - choice c) was made because time domain data is closer than frequency domain data to what is usuallly generated in experimental and field conditions, in addition to being the source of much information. The choice of plane-wave solicitation was arbitrary, so that any other kind (such as source-wave) solicitation could have been employed and would probably lead to the same conclusions as those of the present study;

$D$ - the choice of $L^{2}$ norms is traditional in inverse problem solving. Doing this without 
linearization (e.g., linearization being implemented by the Born approximation) is becoming more and more feasible as the power of computers is increasing. Doing the inversion with regularization is necessary to obtain a unique, stable solution;

$E$ - choice e) of the Simplex algorithm was dictated by the requirement of comparison with many previous inversion studies employing this algorithm, and in particular with the recent study of Buchanan et al. [5]. The Simplex scheme is not optimal in terms of the required number of iterations and function calls, but it is notoriously robust. A gradient-based method would probably lead to the same principal conclusions as those of our study;

$F-H$ - the inverse crime context was chosen to conform with [5] and to illustrate the correct manner of tuning the Simplex algorithm. Non-inverse crime contexts were chosen to better conform to real-world situations in which the values of priors employed in the retrieval model are more-or-less far-removed from their real counterparts. Moreover, the non-inverse crime context leads to cost functions with local minima, instability and severe non-uniqueness which must be dealt-with by various regularization strategies whose efficiencies were evaluated herein.

The answers we obtained to the five questions raised in the sect. 1 are:

1- in the inverse crime situation, it is possible, by applying the zeroth-order (min min cost) regularization, to generate a unique solution to the parameter retrieval problem, provided the cost functional minimization scheme is allowed to do its work to completion (i.e., by permitting the number of function evaluations to be sufficiently-large). The parameters comprising this solution turn out to be, for all practical purposes, the true parameters, i.e., the inverse crime enables an error-free retrieval (i.e., the value of the corresponding cost functional can be of the order of $10^{-18}$, and the cumulative retrieval error of the order of $10^{-9}$ ) of the sought-for parameters;

2- Naturally, the amount of computations necessary to attain this result is all the larger, the less knowledge one has at the outset of the true values of the parameters, but however small be this knowledge, we succeeded in finding the true solution after a sufficient amount of computations, this being true, whatever: the characteristics of the excitation pulse, and the number of sensors as well as their distance from the scattering body;

3 - the inverse crime solution turned out to be very sensitive to the permitted number of maximum function evaluations $(M F E)$. When $M F E$ was of the order of 100 , the inversions did not enable to distinguish a clear-cut set of admissible solutions whereas the choice of $M F E$ of the order of 1000 clearly revealed this set, which tended to be a cluster of points in the parameter space compactly situated around the true target, this being all the more true, the narrower were the starting intervals of the to-be-retrieved parameters;

4- three regularization strategies were employed. The zeroth-order rule, of the min min cost variety and successfully employed in the inverse crime context, turned out to be unsuitable in the non-inverse crime situation. The first-order rule, based on elimination of non-physical retrievals followed by min min cost selection, turned out to be unsuitable for relatively-large discordance between the assumed prior in the the retrieval model and its true counterpart in 
the data simulation model. The second-order rule, based on elimination of retrieved parameters that are outside of their respective starting intervals followed by min min cost selection, led to a unique, stable solution, even for relatively-severe prior discordance. The cumulative error of this solution turned out to be enormously-large (compared to the uncertainty of the prior) for certain prior discordances, in agreement with what was found in [22] (the latter study being concerned with single-parameter retrieval);

5- The non-inverse crime situation was found to be similar to the inverse crime situation by the fact of requiring larger $M F E$ for larger initial uncertainty of the to-be-retrieved parameters, so that to be sure of finding a meaningful retrieval, for large such initial uncertainty, required $M F E$ of the order of 2000 . This number is dependent also on the prior uncertainty, so that relatively- severe prior uncertainty requires wider initial parameter intervals and consequently larger $M F E$.

Further questions that will require answers in subsequent studies:

$i$ - our study (like the one in [22]) presents a grim picture of the possibility of identifying objects (even ones as simple as the homogeneous, circular cylinder considered herein) with an acceptable degree of precision if prior uncertainty is taken into account, so that a natural question is whether there exists a means (e.g., by a different choice of to-be-retrieved parameters to characterize a sample, and/or by including the priors amongst the to-be-retrieved parameters) of reducing the error caused by prior discordance;

$i i$ - our study did not include noise in the synthetic data, so the question is how this noise will affect the quality of the retrievals;

iii- our study employed synthetic data and certain strong hypotheses (notably concerning the location and shape of the object), so the question is how relaxation of these hypotheses and/or the employment of real/experimental data will affect the precision of the retrievals.

\section{References}

[1] Achenbach J.D., Quantitative nondestructive evaluation, Int.J.Solids Struct., 37, 13-27 (1980).

[2] Alleyne D.N., The nondestructive testing of plates using ultrasonic Lamb waves, Phd thesis, University of London, London (1991).

[3] Banks H.T. and Bihari K.L., Modelling and estimating uncertainty in parameter estimation, Inverse Probs., 17, 95-102 (2001).

[4] Banks H.T. and Kunisch K., Estimation Techniques for Distributed Parameter Systems, Birkhauser, Boston (1989).

[5] Buchanan J.L., Gilbert R.P. and Ou M.-J.Y., Recovery of the parameters of cancellous bone by reflection coefficients, Inverse Probs., 27, 125006 (2011). 
[6] Bui H.D., Introduction aux Problèmes Inverses en Mécanique des Matériaux, Eyrolles, Paris (1993).

[7] Byatt D., Coope I. and Price C., 40 Years of the Nelder-Mead algorithm, Talk, University of Canterbury (http://oldweb.cecm.sfu.ca/AAS/coope.pdf) (2003).

[8] Cao D., Beydoun W.B., Singh S.C. and Tarantola A., A simultaneous inversion for background velocity and impedance maps, Geophys., 55, 458-469 (1990).

[9] Chen X., Grzegorczyk T.M., Wu B.-I., Pacheco Jr. J. and Kong J.A., Robust method to retrieve the constitutive effective parameters of metamaterials, Phys.Rev. E, 70, 016608 (2004).

[10] Devlin J. F., A simple and powerful method of parameter estimation using simplex optimization, Ground Water, 32, 323-327 (1994).

[11] Cox H.L.H., Estimation of macro velocity models by wave field extrapolation; Phd thesis, Delft University of Technology, Delft (1991).

[12] Dubois A., Geffrin J.-M. and Belkebir K., Imaging of dielectric cylinders from experimental stepped-frequency data, Appl.Phys.Lett., 88, 164104 (2006).

[13] Gelis C., Virieux J. and Grandjean G., Two-dimensional elastic full waveform inversion using Born and Rytov forumulations in the frequency domain, Geophys.J.Intl., 168, 605$633(2007)$.

[14] Guillermin R., Caractérisation d'objets enfouis dans des sédiments marins par imagerie acoustique, Phd thesis, Université de la Méditerranée Aix-Marseille II, Marseille (2000).

[15] Hadamard, J.S., Lectures on Cauchy's Problem in Linear Partial Differential Equations, Oxford University Press, Oxford (1923).

[16] Huthwaite P. and Simonetti F., High-resolution imaging without iteration: A fast and robust method for breast ultrasound tomography, J.Acoust.Soc.Am., 130, 1721-1734 (2011).

[17] Isakov V., Inverse obstacle problems, Inverse Probs., 25123002 (2009).

[18] Johnson M.L. and Lindsay M. Faunt L.M., Parameter estimation by least-squares methods, in Numerical Computer Methods, Brand L. and Johnson M.L. (eds.), Academic, New York (1992).

[19] Kurrant D. and Fear E., Regional estimation of the dielectric properties of inhomogeneous objects using near-field reflection data, Inverse Probs., 28, 075001 (2012).

[20] Lagarias J., Reeds J., Wright M. and Wright P., Convergence properties of the NelderMead Simplex method in low dimensions, SIAM J.Optim., 9, 112-147 (1998). 
[21] Lavarello Montero R.J., New developments on quantitative imaging using ultrasonic waves, Phd Thesis, University Illinois, Urbana-Champaign (2009).

[22] Lefeuve-Mesgouez G., Mesgouez A., Ogam E., Scotti T. and Wirgin A., Retrieval of the physical properties of an anelastic solid half space from seismic data, J.Appl.Geophys., 88, 70-82 (2013).

[23] McKinnon K., Convergence of the Nelder-Mead Simplex method to a nonstationary point, SIAM J.Optim., 9, 148-158 (1998).

[24] Moghaddam M., Chew W.C. and Oristaglio M., Comparison of the Born iterative method and Tarantola's method for an electromagnetic time-domain inverse problem, Int.J.Imag.Sys.Tech., 3, 318-333 (1991).

[25] Mudry E., Chaumet P.C., Belkebir K. and Sentenac A., Electromagnetic wave imaging of three-dimensional targets using a hybrid iterative inversion method, Inverse Probs., 28, 065007 (2012).

[26] Nelder J. A. and Mead R., A Simplex method for function minimization, The Comput.J., 7, 308-313 (1965).

[27] Niwa Y., Hirose S. and Kitahara M., Application of the boundary integral equation (BIE) method to transient response analysis of inclusions in a half space, Wave Motion, 8, 77-91 (1986).

[28] Ogam E., Caractérisation ultrasonore et vibroacoustique de la santé mécanique des os humains, Phd thesis, Université de Provence Aix-Marseille 1, Marseille (2007).

[29] Ogam E. and Fellah Z.E.A., The direct and inverse problems of an air-saturated poroelastic cylinder submitted to acoustic radiation, AIP Advances, 1, 032174 (2011).

[30] Press W.H., Teukolsky S.A., Vettering W.T. and Flannery B.P., Numerical Recipes in Fortran, Cambridge University Press, Cambridge, 402-406 (1992).

[31] Rayleigh L., The dispersal of light by a dielectric cylinder, Philos.Mag., 36, 365-376 (1918).

[32] Sachse W., Castagnède, Grabec I., Kim K.Y. and Weaver R.L., Recent developments in quantitative ultrasonic NDE of composites, Ultrasonics, 28, 97-104 (1990).

[33] Schmitz G., Ultrasound in medical diagnosis, in Scattering, Pike R. and Sabatier P. (eds.), Academic, San Diego, vol.1, 162-174 (2002).

[34] Scotti T. and Wirgin A., Real-time sonomammography based on the intersecting canonical body approximation of the diffracted wave field, Ultrasonics, 36, 643-652 (1998). 
[35] Scotti T. and Wirgin A., Reconstruction of the three mechanical material constants of a lossy fluid-like cylinder from low-frequency scattered acoustic fields, C.R.Mécanique, $332,717-724$ (2004).

[36] Sebaa N., Fellah Z.E.A., Fellah M., Ogam E., Mitri F.G., Depollier C. and Lauriks W., Application of the Biot model to ultrasound in bone: inverse problem, IEEE Trans.Ultrason.Ferroelec.Freq.Contr., 55, 1516-1523 (2008).

[37] Sun C.-H., Chiu C.-C., Li C.-L. and Huang C.H., Time domain image reconstruction for homogenous dielectric objects by dynamic differential evolution, Electromagnetics, 30, 309-323 (2010).

[38] Tarantola A., A strategy for nonlinear elastic inversion of seismic reflection data, Geophys., 51, 1893-1903 (1986).

[39] Thompson R.B., Laboratory nondestructive evaluation technology for materials characterization, J.Nondestr.Eval., 15, 163-176 (1996).

[40] Tomick J.J., On convergence of the Nelder-Mead simplex algorithm for unconstrained stochastic optimization, Phd thesis, Pennsylvania State University, College Park (1995).,

[41] Virieux J. and Operto S., An overview of full-waveform inversion in exploration geophysics, Geophys., 74, WCC127-WCC152 (2009).

[42] Vugrin K.E., On the effects of noise on parameter identification optimization problems, $\mathrm{PhD}$ thesis, Virginia Polytechnic Institute and State University, Blacksburg (2004).

[43] Wirgin A., Some quasi-analytic and numerical methods for acoustical imaging of complex media, in Wavefield Inversion, Wirgin A. (ed.), Springer, Wien, 241-304 (1999).

[44] Wirgin A., The inverse crime, http://arxiv.org/abs/math-ph/0401050 (2004).

[45] Wirgin A. and Scotti T., Shape reconstruction of a penetrable scattering body via diffracted waves and canonical solutions, Acoustical Imaging, Springer, Berlin, 23, 459464 (1997).

[46] Wiskin J., Borup D.T., Johnson S.A. and Berggren M., Non-linear inverse scattering: High resolution quantitative breast tissue tomography, J.Acoust.Soc.Am., 131, 3802-3813 (2012).

[47] Yeh C.-C. and Yang C.-H., Characterization of mechanical and geometrical properties of a tube with axial and circumferential guided waves, Ultrasonics, 51, 472-479 (2011).

[48] Zhang X., Broschat S.L. and Flynn P.J., A comparison of material classification techniques for ultrasound inverse imaging, J.Acoust.Soc.Am., 111, 457-467 (2002). 